\title{
Digitally Enhanced Software-Defined Radio Receiver Robust to Out-of-Band Interference
}

\author{
Zhiyu Ru, Member, IEEE, Niels A. Moseley, Eric A. M. Klumperink, Senior Member, IEEE, and \\ Bram Nauta, Fellow, IEEE
}

\begin{abstract}
A software-defined radio (SDR) receiver with improved robustness to out-of-band interference $(\mathrm{OBI})$ is presented. Two main challenges are identified for an OBI-robust SDR receiver: out-of-band nonlinearity and harmonic mixing. Voltage gain at RF is avoided, and instead realized at baseband in combination with low-pass filtering to mitigate blockers and improve out-of-band IIP3. Two alternative "iterative" harmonic-rejection (HR) techniques are presented to achieve high HR robust to mismatch: a) an analog two-stage polyphase HR concept, which enhances the HR to more than $60 \mathrm{~dB}$; b) a digital adaptive interference cancelling (AIC) technique, which can suppress one dominating harmonic by at least $80 \mathrm{~dB}$. An accurate multiphase clock generator is presented for a mismatch-robust $H R$. A proof-of-concept receiver is implemented in $65 \mathrm{~nm}$ CMOS. Measurements show $34 \mathrm{~dB}$ gain, $4 \mathrm{~dB} \mathrm{NF}$, and $+3.5 \mathrm{dBm}$ in-band IIP3 while the out-of-band IIP3 is $+16 \mathbf{d B m}$ without fine tuning. The measured RF bandwidth is up to $6 \mathrm{GHz}$ and the 8-phase LO works up to $0.9 \mathrm{GHz}$ (master clock up to $7.2 \mathrm{GHz}$ ). At $0.8 \mathrm{GHz}$ $L O$, the analog two-stage polyphase HR achieves a second to sixth order $H R>60 \mathrm{~dB}$ over 40 chips, while the digital AIC technique achieves $H R>80 \mathrm{~dB}$ for the dominating harmonic. The total power consumption is $50 \mathrm{~mA}$ from a $1.2 \mathrm{~V}$ supply.
\end{abstract}

Index Terms-Adaptive interference cancellation, adaptive signal processing, baseband processing, blocker, blocker filtering, CMOS, cross-correlation, digitally assisted, digitally enhanced, harmonic mixing, harmonic rejection, interference mitigation, linearity, LMS, low-noise amplifier (LNA), low-noise transconductance amplifier (LNTA), mismatch, multiphase, multiphase clock, nonlinearity, out-of-band interference, passive mixer, polyphase, receiver, robust receiver, SAW-less, software radio (SWR), software-defined radio (SDR), switching mixer, wideband receiver.

\section{INTRODUCTION}

$\mathbf{S}$ OFTWARE-DEFINED RADIO (SDR) concepts have recently drawn considerable academic interest and increasingly also industrial interest. Limiting our discussion to $\mathrm{RF}$ transceivers, most work focuses on integrating the functionality of multiple dedicated narrowband radios into one radio, which is reconfigurable by software [1], [2]. This is hoped to bring cost and size reductions while supporting an ever increasing set of communication standards in a single device. The SDR concepts might also allow field upgradable radios to accommodate emerging standards and become an enabler

Manuscript received April 27, 2009; revised August 10, 2009. Current version published December 11, 2009. This paper was approved by Guest Editor Nikolaus Klemmer. This work was supported by Freeband Communications and NXP Semiconductors.

The authors are with the Department of Electrical Engineering, University of Twente, Enschede 7500 AE, The Netherlands (e-mail: z.ru@ewi.utwente.nl; n.a.moseley@alumnus.utwente.nl).

Digital Object Identifier 10.1109/JSSC.2009.2032272 for cognitive radio applications, to improve the efficiency of utilizing the scarce spectrum resources.

To support the reception of different radio standards, a wideband radio receiver seems an obvious solution. Some wideband receivers have been reported, e.g., for wideband TV receivers [3], [4], ultra-wideband receivers [5], [6], and SDR applications [1], [2]. However, wideband receivers are not only wideband to desired signals but also wideband to undesired interference.

Traditional wireless standards use dedicated radio bands, so that in-band interference (IBI) can be distinguished from out-ofband interference (OBI). For a SDR aiming at covering arbitrary frequencies, the definition of IBI and OBI may become fuzzy. Still, we will use the terms IBI and OBI in this paper as: 1) current SDR receivers often aim at covering multiple traditional radio standards which have clear band definitions; 2) even if this is not the case, e.g., for cognitive radio, a SDR still aims at implementing selectivity, i.e., receive a signal for which baseband bandwidth is much smaller than $f_{R F}$. In the latter case OBI can be interpreted as "out-of-baseband interference".

For popular mobile communication applications, the IBI can be as strong as -30 to $-20 \mathrm{dBm}$ while the OBI can be as strong as -10 to $0 \mathrm{dBm}$ [7]. An RF band-selection filter is often employed to suppress OBI to below the IBI level, requiring high quality factor and sharp roll-off. These filters are difficult to integrate on-chip and are often dedicated to one specific band. In a SDR receiver, the dedicated RF filter is undesired owing to its poor flexibility. State-of-the-art multi-band receivers [8], [9] use multiple dedicated RF filters in parallel, which increases size and cost for every band that is added. This paper aims at improving the robustness of a radio receiver to OBI in order to relax the requirement on RF filters, exploiting fully integrated analog and digitally enhanced mixed-signal techniques.

At least two mechanisms generate in-band distortion due to OBI: 1) nonlinearity related mixing of strong OBI via, e.g., intermodulation or cross-modulation; 2) harmonic mixing of interferers with LO harmonics due to hard-switching mixers and/or the use of digital $\mathrm{LO}$ waveforms. We will explain these two mechanisms briefly below as well as review the state-ofthe-art solutions for these problems.

\section{A. Out-of-Band Nonlinearity}

Nonlinearity may generate intermodulation and harmonic distortion falling on top of the desired signal, or may desensitize a receiver due to blockers and produce cross modulation [10]. Without sufficient RF band-selection filtering, the out-of-band linearity can become the bottleneck since OBI is much stronger than IBI. A wideband LNA as used in [1] and [2] amplifies the desired signal and undesired wideband interference with equal 
gain. A low voltage gain of $6 \mathrm{~dB}$ can already clip a $0 \mathrm{dBm}$ blocker to a $1.2 \mathrm{~V}$ supply. The amplified interference also challenges the nonlinear output impedance of an LNA and the linearity of a next-stage mixer.

LNA linearization techniques have been proposed [11], [12] to achieve an IIP3 in excess of $+15 \mathrm{dBm}$ but have drawbacks [13]: 1) they often rely on two nonlinearity mechanisms that compensate each other but do not automatically match, so that some kind of fine tuning is needed, compromising robustness to process spread; 2) they mostly rely on modeling of the weakly nonlinear region so that high IIP3 is only achieved for low input two-tone power while limited or no benefit for strong interference.

Recently, a blocker filtering technique has been presented [14], achieved by means of an auxiliary feedforward path, which conducts the undesired interferers and suppresses them by subtracting them from the main signal path at the output of LNA. However it comes with some drawbacks: 1) significant cost arises in terms of noise and power consumption in that auxiliary path; 2) the blocker filtering effect relies on the matching between the main path and the auxiliary path. We will see later in Section II that equivalent functionality can be achieved with much simpler hardware, i.e., without requiring additional signal path.

\section{B. Harmonic Mixing}

Linear time-variant behavior in a hard-switching mixer, or equivalently multiplication with a square wave, not only downconverts the desired signal but also interference around LO harmonics. This harmonic mixing is of much less concern in narrowband receivers, relying on RF band-selection filters. The 8-phase harmonic-rejection (HR) mixers as described in [15] can suppress RF signals around second to sixth LO harmonics but amplitude and phase mismatches limit the achievable HR ratio typically to 30 -to-40 dB [2]-[4], [16]. However, a quick calculation shows that much more rejection is needed: if we want to bring harmonic responses down to the noise floor, e.g., $-100 \mathrm{dBm}$ in $10 \mathrm{MHz}$ for $N F=4 \mathrm{~dB}$, and cope with interferers of -40 to $0 \mathrm{dBm}$, a HR ratio of 60 to $100 \mathrm{~dB}$ is needed. State-of-the-art wideband TV tuners rely on RF tracking filters together with HR mixers [3], [4] to guarantee more than $65 \mathrm{~dB}$ HR ratio. We aim at removing such tracking filters or at least relaxing their requirements by making HR mixers more robust to mismatch.

\section{Contribution of This Paper}

Both out-of-band nonlinearity and harmonic mixing can severely degrade signal-to-distortion ratio. ${ }^{1}$ Therefore, in our view a practical SDR should not just be a wideband receiver, but also have enhanced out-of-band linearity and enhanced harmonic rejection. This paper will propose an architecture to improve the wideband receiver's linearity, especially its IIP3 for OBI and its tolerance to blockers. Moreover, to dramatically improve HR performance, two alternative HR techniques are proposed: 1) a two-stage polyphase HR technique implemented

\footnotetext{
${ }^{1}$ Signal-to-distortion ratio is so important to software-defined radio that it can be viewed as another interpretation of "SDR".
}

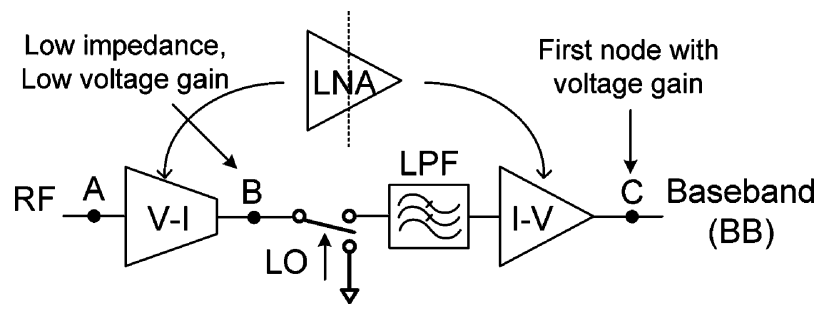

Fig. 1. Conceptual diagram of the low-pass blocker filtering.

purely in the analog domain [17], [18]; 2) a mixed-signal technique exploiting digital adaptive interference cancelling (AIC) [19]. Both improve HR by rejecting harmonics in two successive steps ("iterative"), and both share the same 8-phase RF-to-baseband downconverter as a first HR stage. Compared to [17]-[19], we greatly extend the analysis and show additional experimental results. Compared to [20], this work derives the interference estimate in another way, presents measurements and achieves better performance due to the better interference estimate.

The rest of the paper is organized as follows. Section II introduces a technique using low-pass filtering to mitigate blockers and improve out-of-band IIP3. Section III proposes a two-stage polyphase HR concept to improve amplitude accuracy obtaining high HR robust to mismatch. To improve both amplitude and phase accuracy, a digitally enhanced HR technique using AIC is presented in Section IV. The implementations of the analog front-end and the digital back-end are discussed in Sections $\mathrm{V}$ and VI respectively. The experimental results are presented in Section VII with a comparison of analog and digital HR techniques as well as benchmarking to other work. The conclusions are drawn in Section VIII.

\section{LOW-PASS BLOCKER FILTERING}

Traditionally, narrowband receiver front-ends use LNAmixer combinations which can deliver good enough linearity, typically an IIP3 $<0 \mathrm{dBm}$, for in-band (IB) interference while an RF band-selection filter takes care of out-of-band (OB) interference. However, in a wideband receiver, since OBI is much stronger than IBI, the required OB IIP3 is much higher than the required IB IIP3 and even desensitization can occur due to strong OB blockers. Therefore, frequency selective amplification or attenuation is desired. Tunable bandpass filtering (BPF) is in principle a solution, but it is difficult to provide sufficient selectivity and tunability simultaneously with good noise and linearity, using CMOS on-chip filters. Here we approach the problem from another angle.

\section{A. Concept}

To guarantee low NF, we need amplification early in the receiver chain. Voltage amplification in an LNA is usually realized via V-I conversion using, e.g., the transconductance of a transistor, followed by I-V conversion via some impedance or transimpedance. We can separate the two functional blocks, V-I and I-V, and insert a passive zero-IF mixer and a low-pass filter (LPF) in between, as shown in Fig. 1. The LPF drawn is conceptually current-in current-out and internally with no voltage swing. However in practice, the functions of the LPF and the 
$\mathrm{I}-\mathrm{V}$ conversion can be merged by using a frequency-dependent impedance, such as a parallel $\mathrm{R}$ and $\mathrm{C}$.

It is crucial to present a low impedance over a wide band to the output of V-I block, i.e., node B, so that little voltage gain occurs before filtering, leading to less distortion in the mixer and the nonlinear output impedance of the V-I block. ${ }^{2}$ Therefore, the first voltage gain occurs only at baseband after low pass filtering, which provides selectivity to mitigate OBI.

To quantify the blocker filtering effect, we may compare the $1 \mathrm{~dB}$ compression point $\left(P_{1 \mathrm{~dB}}\right)$ for desired signals to the $1 \mathrm{~dB}$ desensitization point $\left(B_{1 \mathrm{~dB}}\right)$ for blockers, ${ }^{3}$ both input-referred. Assume a third order Taylor series for nonlinearity with $\alpha_{1}$ and $\alpha_{3}$ for the first and third order coefficients respectively. Without any blocker filtering, it can be derived from [10] that $P_{1 \mathrm{~dB}}=$ $10 \cdot \log \left(0.145 \cdot\left|\alpha_{1} / \alpha_{3}\right|\right)$ and $B_{1 \mathrm{~dB}}=10 \cdot \log \left(0.0725 \cdot\left|\alpha_{1} / \alpha_{3}\right|\right)$, if both in amplitude. Therefore, $B_{1 \mathrm{~dB}}$ can be calculated based on $P_{1 \mathrm{~dB}}$, and if without blocker filtering, $B_{1 \mathrm{~dB}}=\left(P_{1 \mathrm{~dB}}-3 \mathrm{~dB}\right)$.

The LPF in Fig. 1 can mitigate blockers, and its bandwidth (BW) and order (n) determines the blocker filtering effect. If desensitization happens after I-V conversion, which is often the case due to a high voltage gain and limited voltage headroom, the suppression of blockers in $\mathrm{dB}$ by the LPF corresponds to the improvement of $B_{1 \mathrm{~dB}}$.

However, for a wideband receiver the situation is more complicated, as one RF-blocker can be downconverted by different LO harmonics. For instance, a square-wave LO of $400 \mathrm{MHz}$ converts a $1250 \mathrm{MHz} \mathrm{RF}$ signal to $850 \mathrm{MHz}$ and 50 $\mathrm{MHz}$ via the first and third harmonic of the LO,respectively. The strongest downconverted signal depends on the blocker frequency $\left(f_{B}\right)$ and the $\mathrm{LO}$ frequency $\left(f_{\mathrm{LO}}\right)$, i.e., which LO harmonic the blocker is closer to. Also it depends on the relative gain of the $m^{\text {th }}$ harmonic compared to the fundamental (first) harmonic, i.e., the $m^{\text {th }}$ harmonic rejection ratio $\left(H R_{m}\right)$.

Assume for simplicity that one blocker component dominates after downconversion and determines $B_{1 \mathrm{~dB}}$. If $\left|f_{B}-m \cdot f_{\mathrm{LO}}\right| \leq$ $\mathrm{BW}$, i.e., the blocker is within the LPF BW after downconversion by the $m^{\text {th }}$ harmonic, we find

$$
B_{1 \mathrm{~dB}} \approx\left(P_{1 \mathrm{~dB}}-3 \mathrm{~dB}\right)+\min \left[H R_{m}\right] .
$$

If $\left|f_{B}-m \cdot f_{\mathrm{LO}}\right|>B W$, i.e., the blocker is outside the LPF BW after downconversion by the $m^{\text {th }}$ harmonic, assuming an asymptotic filter characteristic, we find

$$
\begin{aligned}
B_{1 \mathrm{~dB}} \approx & \left(P_{1 \mathrm{~dB}}-3 \mathrm{~dB}\right) \\
& +\min \left[n \cdot 20 \log \left(\frac{\left|f_{B}-m \cdot f_{\mathrm{LO}}\right|}{\mathrm{BW}}\right)+H R_{m}\right] .
\end{aligned}
$$

From (2) we can expect smaller bandwidth (BW) and higher order (n) of the LPF gives higher $B_{1 \mathrm{~dB}}$, if $f_{B}, f_{\mathrm{LO}}$ and $H R_{m}$ are fixed. Besides, we can also improve $B_{1 \mathrm{~dB}}$ via improving $P_{1 \mathrm{~dB}}$, e.g., if compression happens at the receiver output, a lower receiver voltage gain or a larger output voltage headroom can improve the input-referred $P_{1 \mathrm{~dB}}$, and hence a higher $B_{1 \mathrm{~dB}}$.

\footnotetext{
${ }^{2}$ Another motivation for low impedance at RF nodes is to widen the receiver's RF bandwidth as exploited in [5].

${ }^{3} P_{1 \mathrm{~dB}}$ thus defines the desired input signal power at which the receiver gain drops by $1 \mathrm{~dB}$ without applying blockers, while $B_{1 \mathrm{~dB}}$ defines the undesired input interference (single-tone blocker) power where the receiver gain drops by $1 \mathrm{~dB}$.
}

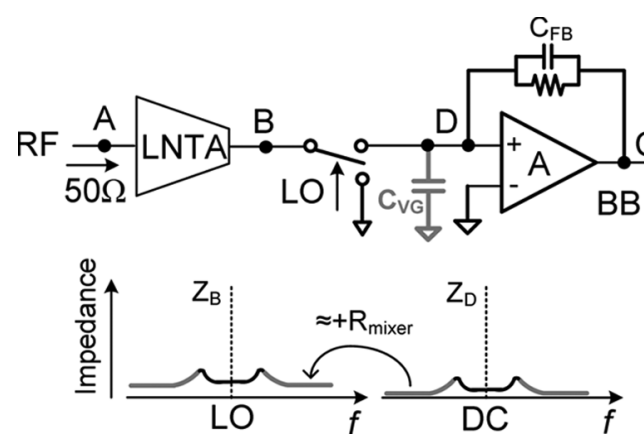

Fig. 2. Realization of the low-pass blocker filtering and illustration of impedance transfer effect.

The LPF can help to relax the OB linearity of the I-V conversion, however not for the V-I conversion. Therefore, the maximum achievable $B_{1 \mathrm{~dB}}$ is ultimately limited by the $P_{1 \mathrm{~dB}}$ of the V-I conversion minus $3 \mathrm{~dB}$. Thus, linearity of the V-I conversion is very important and we will return to that point in Section V-A. Via a similar mechanism, the OB IIP3 can also be enhanced compared to the IB IIP3.

\section{B. Realization}

A specific realization of the general concept (Fig. 1) is presented in Fig. 2. Zero-IF receivers commonly use an LNA followed by a mixer with current output loaded by a LPF to suppress interference. We carry this approach one step further by entirely removing the voltage-gain LNA before the mixer and instead use a Low Noise Transconductance Amplifier (LNTA) as the first RF stage for the $\mathrm{V}$-I conversion with input impedance matching. As mentioned before, maintaining a low impedance at node B over a wide band is important. This can be realized by using low-ohmic switches in the passive mixers followed by transimpedance amplifiers (TIA) built via negative feedback around operational transconductance amplifier (OTA). The feedback network consists of $\mathrm{R} \& \mathrm{C}$ in parallel to form a LPF. At high frequency, the feedback loop gain drops so the virtual-ground impedance rises. By putting a capacitor $C_{\mathrm{VG}}$ to ground or across the differential virtual-ground nodes, the impedance at high frequency is reduced. Both $C_{\mathrm{VG}}$ and $C_{\mathrm{FB}}$ contribute to the total LPF function.

Fig. 2 also shows, qualitatively, the impedance relationship between node $\mathrm{B}\left(Z_{B}\right)$ and node $\mathrm{D}\left(Z_{D}\right)$, i.e., $Z_{B}$ is roughly equal to a certain scaling factor times $Z_{D}$ plus the mixer switch-on resistance $\left(R_{\text {mixer }}\right)$ and shifted in frequency. Applying an RF current input, it can be derived [21] that, for an $\mathrm{N}$-phase mixer driven by $1 / \mathrm{N}$-duty-cycle (non-overlapping) $\mathrm{LO}$, the impedance $Z_{B}$ at an RF around $m^{\text {th }}$-LO-harmonic frequency $(m=1,2,3 \ldots)$, i.e., with an offset frequency $\Delta f$ from $m \cdot f_{\mathrm{LO}}\left(|\Delta f| \leq f_{\mathrm{LO}} / 2\right)$, can be written as

$Z_{B}\left(m \cdot f_{\mathrm{LO}}+\Delta f\right) \approx R_{\text {mixer }}+\frac{N}{m^{2} \cdot \pi^{2}} \cdot \sin ^{2}\left(\frac{m \cdot \pi}{N}\right) \cdot Z_{D}(\Delta f)$.

Please note that (3) holds given that $Z_{D}$ presents strong filtering effect, e.g., a pole at a much lower frequency than $f_{\mathrm{LO}}$, which is normally the case for a downconversion mixer. Consider $m=$ 1: for $N=2$ or 4 the coefficient of $Z_{D}$ is about 0.2 , and for 


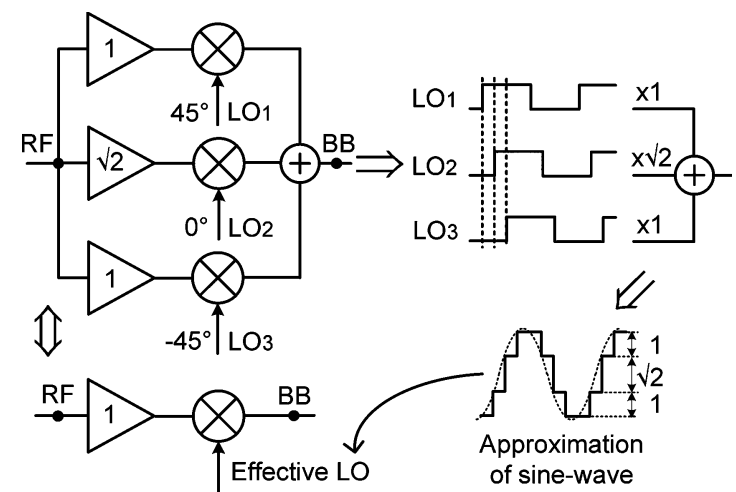

(a)
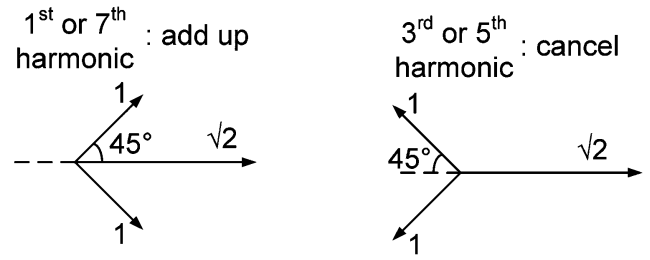

(b)

Fig. 3. (a) Block diagram of a traditional HR mixer, and (b) its vector diagram.

$N=8$, it is about 0.12 , showing $R_{\text {mixer }}$ actually plays a much larger role in determining $Z_{B}$. For $m>1$, the coefficient of $Z_{D}$ is even smaller.

Besides delivering low impedance, this topology (Fig. 2) can also bring two other advantages exploited in some narrowband receivers [22]-[24]: 1) good in-band linearity in the I-V conversion due to the negative feedback; 2) low 1/f noise from the mixer switches working in the linear region which carry little DC current. This work [17] exploits this topology in a wideband receiver to enhance out-of-band linearity. If the LPF suppresses the OBI well, the main contributor to the OB nonlinearity will come from the V-I conversion of the LNTA, which can be quite linear as we will see later.

Although voltage amplification is avoided at RF, if the transconductance of LNTA is big, the receiver-input-referred noise of the following stages, i.e., mixer and TIA, can be relatively small, so that the overall receiver NF can still be good and dominated by LNTA itself. As an example, the whole receiver in [24] achieves an NF of $2.2 \mathrm{~dB}$ based on a similar topology but in a narrowband configuration.

\section{Two-Stage Polyphase HaRmonic ReJection}

The low-pass blocker filtering technique presented in the previous section acts after mixing, so it cannot prevent the harmonic mixing already occurring in the mixer stage. It is known that using balanced LO can suppress all even-order harmonics. To also suppress odd-order harmonics, harmonic-rejection (HR) mixers using multi-phase square-wave LOs driving parallel operating mixers have been proposed before [15], [16]. Fig. 3(a) shows an example, where the weighted current outputs add up to approximate mixing with a sine-wave LO. The combination of an amplitude ratio of $1: \sqrt{2}: 1$ and an 8-phase $\mathrm{LO}^{4}$ (equidistant $45^{\circ}$ ) can reject the third and fifth harmonics, as shown in the

\footnotetext{
${ }^{4}$ Using more LO phases can reject more harmonics, but it complicates LO generation.
}

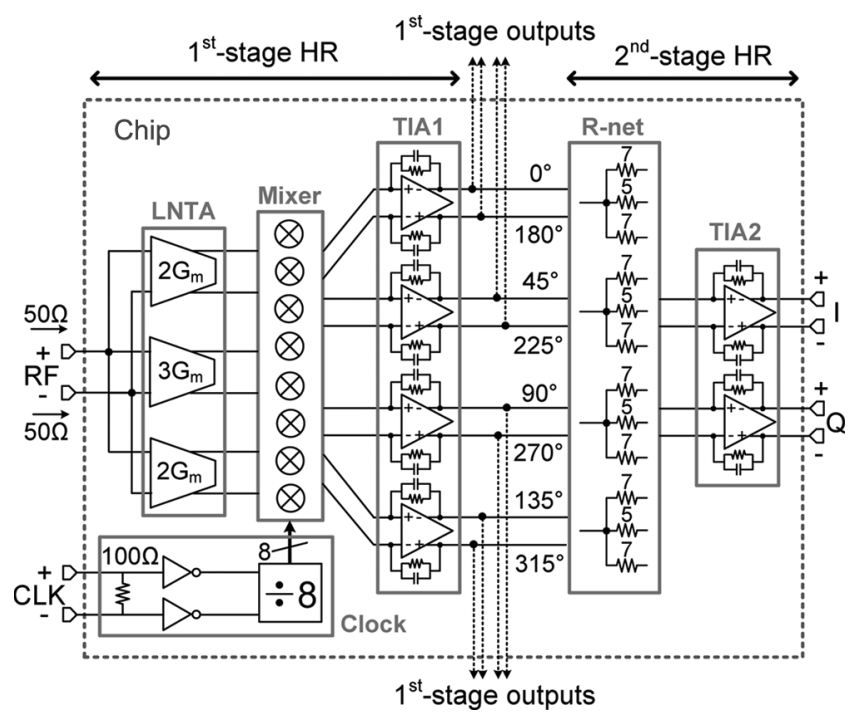

Fig. 4. Chip block diagram implementing the two-stage polyphase HR and the low-pass blocker filtering.

vector diagram of Fig. 3(b). The seventh harmonic is not rejected and still needs to be removed by filtering, but the filter requirement is strongly relaxed compared to the case of a normal I/Q mixer whose first un-rejected harmonic is the third order. However, the achievable HR ratio is limited by the accuracy of the amplitude ratios and the LO phases.

To achieve high HR ratio we need to accurately implement the desired weighting ratios, in this case the irrational ratio $1: \sqrt{2}$ accurately on chip. There are at least two challenges here: 1) realizing the right nominal (average) ratio; 2) keeping random variations due to mismatch small enough. To address these issues we propose a two-stage polyphase HR concept (see Fig. 4) in which two-stage iterative weighting and summing results in much higher HR than traditional HR mixers with only one stage. We will show that this iterative weighting results in a small product of relative errors for random variations, whereas the use of suitably chosen integer ratios results in sufficient accuracy to achieve a HR well above $60 \mathrm{~dB}$.

\section{A. Block Diagram}

Fig. 4 shows the block diagram of the two-stage polyphase HR system, implemented on chip. The irrational ratio $1: \sqrt{2}: 1$ is realized in two iterative steps with integer ratios: a first step with $2: 3: 2$ and a second step with 5:7:5. The first-stage weighting is realized via 7 unit-LNTAs interconnected in 3 parallel groups to form the 2:3:2 ratio. The second-stage weighting is realized via a baseband resistor network "R-net" between the TIA1 and TIA2 stages. The 5:7:5 amplitude ratio corresponds to the 7:5:7 resistance ratio. The passive mixer array is driven by 8-phase 1/8-duty-cycle (non-overlapping) LO. Via the combination of the LNTA, mixer and TIA with LPF, the first voltage gain occurs at baseband after LPF for good OB linearity. Since harmonics can be as strong as blockers, it is important to have significant HR before the first voltage gain, especially because the antiblocker filtering does not reduce harmonic images close to harmonics of the LO, as shown in (1). The additional more accurate HR follows in the second stage, aiming to bring residual harmonic images below the noise floor. 


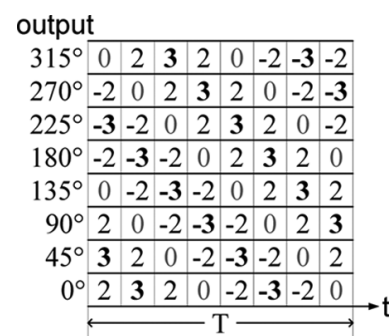

Fig. 5. Weighting factors for the first-stage HR outputs versus time.

\begin{tabular}{|c|c|c|c|c|c|c|c|}
\hline [2 & 3 & 2 & 0 & -2 & -3 & -2 & $0] \cdot 5$ \\
\hline$+[0$ & 2 & 3 & 2 & 0 & -2 & -3 & $-2] \cdot 7$ \\
\hline$+[-2$ & 0 & 2 & 3 & 2 & 0 & -2 & $-3] \cdot 5$ \\
\hline
\end{tabular}

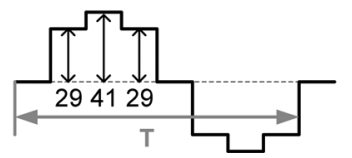

Fig. 6. Approximation of $1: \sqrt{2}: 1$ as $29: 41: 29$ via integer ratios.

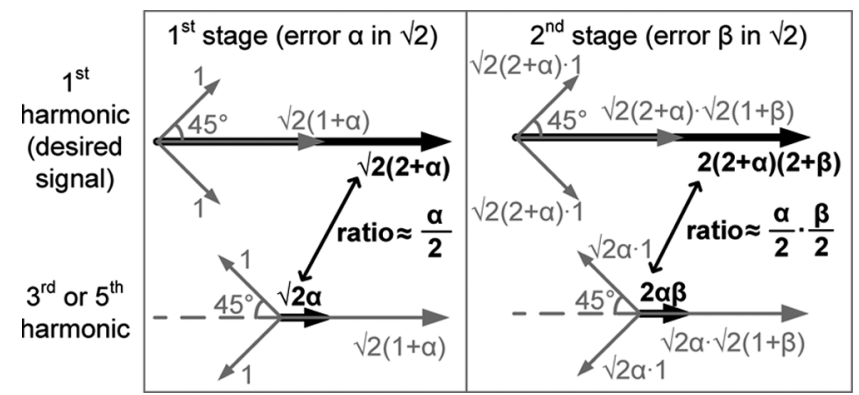

Fig. 7. Error reduction principle in the two-stage polyphase HR (error $\alpha / 2$ becomes a much smaller product of errors: $\alpha \beta / 4$ ).

\section{B. Working Principle}

We will now show how we accurately approximate $1: \sqrt{2}: 1$ via 2:3:2 and 5:7:5. A key point is that the output of the TIA1 stage has $8 \mathrm{IF}$-outputs with equidistant phases, i.e., $0^{\circ}$ to $315^{\circ}$ with $45^{\circ}$ step, instead of the conventional 4 phases, i.e., quadrature. This enables iterative HR by adding a second stage. Fig. 5 shows the weighting factor for the 8 outputs of the first-stage HR versus time ( $t$ ) for one complete period of the $\mathrm{LO}$ (T). If we weight and sum three adjacent-phase outputs of the first-stage HR via the second-stage weighting factors 5:7:5, as shown in Fig. 6, we find 29:41:29. The ratio 41:29 is equal to 1.4138 , which represents only a $0.028 \%$ error from $\sqrt{2}$. This amplitude error corresponds to a $\mathrm{HR}$ ratio of more than $77 \mathrm{~dB}$, if no phase error.

The two-stage polyphase HR not only can approximate $1: \sqrt{2}: 1$ very closely, but it is also robust to amplitude mismatch, as illustrated in Fig. 7 via vector diagrams of the two stages. It shows how, for the desired signal, polyphase contributions from three paths add up, while for the third and fifth harmonics, they cancel nominally. Assume now that the error in realizing $\sqrt{2}$ dominates and model it as a relative error $\beta$ for the first stage and $\alpha$ for the second stage. Also for simplicity, assume that the desired signal and the third and fifth harmonics

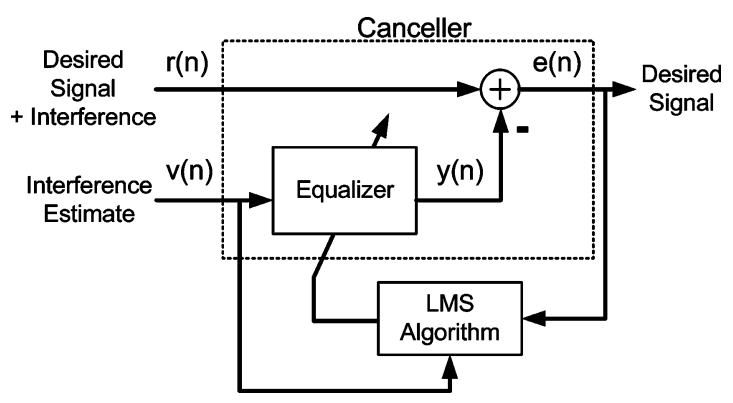

Fig. 8. A block diagram showing the concept of adaptive interference cancelling.

are equally strong at the receiver input and neglect the relative strength of different LO harmonics due to a certain LO duty cycle. After the first stage, the desired signal is multiplied by $\sqrt{2} \cdot(2+\alpha)$ and the third and fifth harmonics by $\sqrt{2} \cdot \alpha$, leading to a relative error (interference-to-signal ratio) of $\alpha / 2$ if $\alpha \ll 2$. For the second stage the same derivation holds. As the two stages are cascaded, the product of the gains determines the result, i.e., the total gain for the desired signal becomes $[\sqrt{2} \cdot(2+\alpha)] \cdot[\sqrt{2} \cdot(2+\beta)]$ and for the third and fifth harmonics it is $[\sqrt{2} \cdot \alpha] \cdot[\sqrt{2} \cdot \beta]$. This renders a total relative error (interference-to-signal ratio) of

$$
\frac{2 \alpha \beta}{2 \cdot(2+\alpha) \cdot(2+\beta)} \approx \frac{\alpha}{2} \cdot \frac{\beta}{2}
$$

if $\alpha \ll 2$ and $\beta \ll 2$. Therefore, the total relative error is the product of the relative errors for the two stages, $\alpha / 2$ and $\beta / 2$. If the second stage has an error $\beta=1 \%$, ideally this improves HR by $(\beta / 2)^{-1}$, i.e., $46 \mathrm{~dB}$, which has also been confirmed by simulation.

Please note that the product of errors, as shown in (4), holds for both third and fifth harmonics. Moreover, it not just works for mismatch induced errors but for any amplitude errors, e.g., errors introduced by parasitic capacitance or finite LNTA output impedance.

Theoretically, more than two stages can achieve even better amplitude accuracy, but practically phase accuracy will often dominate. To also address the phase error, next we will propose an alternative HR concept that exploits digital techniques.

\section{Digitally Enhanced HaRmonic Rejection}

Even for the concept proposed in the previous section, the HR performance can still be limited by the amplitude and especially phase mismatches between the paths. In this section, we propose a digitally enhanced HR architecture exploiting digital adaptive interference cancelling (AIC). Simply put, this concept adapts an estimate of the third or fifth order harmonic image in such a way that after subtraction from the received signal the HR ratio is increased.

The AIC concept is shown in Fig. 8: the interference estimate, $\mathrm{v}(\mathrm{n})$, is aligned (in phase and amplitude) with the interference in the received signal, $r(n)$, by an adaptive digital equalizer. Thus, the equalizer removes the amplitude and phase differences of the interference between $v(n)$ and $r(n)$. The equalized interference estimate is subtracted from the received signal, which cancels the interference and produces the output signal, e(n). 


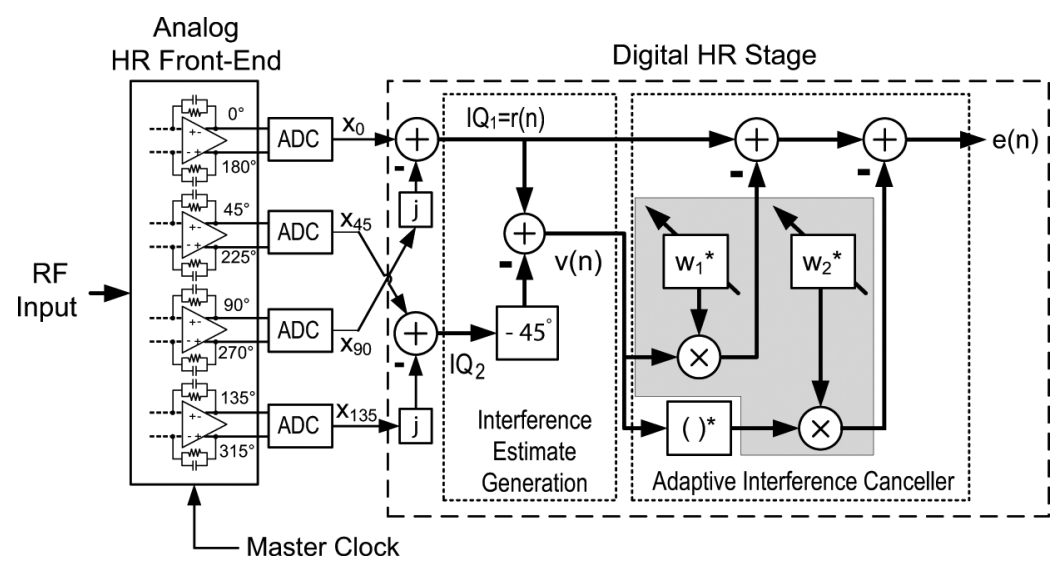

Fig. 9. A system-level block diagram of the analog front-end, the interference estimate generation and the AIC. The equalizer of the AIC is shown in grey.

Fig. 9 shows a system-level block diagram of the proposed system. The analog front-end used is identical to the first stage of the two-stage analog HR architecture proposed earlier. It produces four fully differential signals, which are converted into the digital domain using four $\mathrm{A} / \mathrm{D}$ converters, to form signals $x_{0}$, $x_{45}, x_{90}$ and $x_{135}$. The HR of the analog down-mixer, typically in the range of 30 to $40 \mathrm{~dB}$, reduces the required dynamic range of the aforementioned $\mathrm{A} / \mathrm{D}$ converters.

Two complex-valued IQ pairs are formed using the four realvalued baseband signals:

$$
\begin{aligned}
& I Q_{1}(n)=x_{0}(n)-j \cdot x_{90}(n) \\
& I Q_{2}(n)=x_{45}(n)-j \cdot x_{135}(n)
\end{aligned}
$$

where $I Q_{1}$ can be considered as the received signal and $I Q_{2}$ is an additional I/Q pair, needed to generate the interference estimate.

The baseband signals, $x_{0}, x_{45}, x_{90}$ and $x_{135}$, produced by the analog front-end are subject to component mismatches and LO timing errors, which cause amplitude and phase uncertainty. As a result, the amplitude and phase difference between the received signal, $r(n)$, and the interference estimate, $v(n)$, are subject to this uncertainty.

Perfect cancelling of the interference requires two conditions to be met: first, the interference estimate must be a perfect representation of the interference and second, the amplitude and phase difference between the interference estimate and the interference in the received signal must be completely removed by the equalizer.

Given the above, the equalizer must be adaptive to be able to cope with the uncertainty in the phase and amplitude in order to obtain the maximum amount of interference canceling.

The equalizer consists of two single-tap FIR filters, which are formed by the complex coefficients, $w_{1}, w_{2}$ and the two associated multipliers shown in the grey portion of Fig. 9. The coefficients are adapted by applying the power-normalized LMS algorithm [25].

For the single interferer case (only a third or fifth order harmonic image is present), the signal-to-interference ratio (SIR)
TABLE I

THE Normalized (TO THE Desired Signal) RF-TO-BASEband TRANSFER CHARACTERISTICS OF $I Q_{1}, I Q_{2}$ AND THE INTERFERENCE ESTIMATE v $(\mathbf{n})$

\begin{tabular}{|c|c|c|c|c|c|c|}
\hline \multirow{2}{*}{ Signal } & \multicolumn{2}{|c|}{$I Q_{1}$} & \multicolumn{2}{c|}{$I Q_{2}$} & \multicolumn{2}{c|}{$\begin{array}{c}\text { Interference } \\
\text { estimate, } v(n)\end{array}$} \\
\cline { 2 - 7 } & Gain & Phase & Gain & Phase & Gain & Phase \\
\hline Desired & 1.000 & $0^{\circ}$ & 1.000 & $45^{\circ}$ & 0.000 & $0^{\circ}$ \\
\hline $3^{\text {rd }}$ & 0.024 & $-90.0^{\circ}$ & 0.024 & $135^{\circ}$ & 0.048 & $-90.0^{\circ}$ \\
\hline $5^{\text {th }}$ & 0.014 & $90^{\circ}$ & 0.014 & $-45^{\circ}$ & 0.028 & $90.0^{\circ}$ \\
\hline
\end{tabular}

at the output, e(n), of the digital AIC stage is determined by the inverse SIR of the interference estimate, $v(n)$ [26]:

$$
S I R_{e(n)} \approx \frac{1}{S I R_{v(n)}} .
$$

To maximize the SIR at the output of the canceller, the SIR of the interference estimate must be minimized. Therefore, the aim is to generate an interference estimate that contains the least amount of desired signal energy and the maximum amount of harmonic image energy.

\section{A. Generating the Interference Estimate}

The analog baseband outputs of the front-end, $x_{0}, x_{45}, x_{90}$ and $x_{135}$, are formed by 8 -phase $1 / 8$-period-shifted LO waveforms that approximate a sinusoid, as explained in Section III. An N/8-period time shift results in a $N \cdot 45^{\circ}$ phase shift for the desired signal and three and five times as much for the third and fifth harmonic images. 5 This property is exploited in the generation of the interference estimate.

Considering only the relatively large (6\%) approximation error of $1: \sqrt{2}: 1$ by $2: 3: 2$ (weighting ratio of the three LNTAs), the theoretical RF-to-baseband gain and rotation of the desired and third and fifth order signals are given in Table I. For instance, it shows that the third harmonic image is attenuated by $-20 \cdot \log _{10}(0.024)=32.4 \mathrm{~dB}$, with respect to the desired signal.

${ }^{5} \mathrm{~A}$ time-shift is a linear phase operation. Thus, the resulting phase shift scales linearly with frequency. 
The data for $I Q_{1}$ and $I Q_{2}$ in Table I can be derived using the mixer modeling technique used in [20], which uses the Fourier series of the effective LO waveforms and the LNTA weighting ratio. Note that the phase and amplitude relations between $I Q_{1}$ and $I Q_{2}$ are independent of the actual $R F$ signals, i.e., modulation schemes.

By examining Table I, it follows that the interference estimate, $\mathrm{v}(\mathrm{n})$, can be generated by a $-45^{\circ}$ rotation of $I Q_{2}$, which aligns the desired signal with respect to $I Q_{1}$. Subtracting the rotated $I Q_{2}$, i.e., $I Q_{2}^{\prime}$, from $I Q_{1}$ results in the cancelling of the desired signal but leaves the interference:

$$
v(n)=I Q_{1}(n)-\underbrace{I Q_{2}(n) \cdot \exp \left(-j \cdot \pi \cdot \frac{45}{180}\right)}_{I Q_{2}^{\prime}(n)} .
$$

The resulting signal components in the interference estimate, also shown in Table I, can be derived using (6). For instance, it shows that the third harmonic image is attenuated by -20 . $\log _{10}(0.048)=26.4 \mathrm{~dB}$. This attenuation is solely due to the analog HR front-end and the application of (6). The third harmonic image, in the interference estimate, is $6 \mathrm{~dB}$ stronger compared to $I Q_{1}$ or $I Q_{2}$ owing to a doubling of its amplitude by (6). This also holds for the fifth harmonic image. In addition, the desired signal is completely cancelled, despite the $6 \%$ error in $1: \sqrt{2}: 1$. Thus, in theory, $\mathrm{v}(\mathrm{n})$ can be a good interference estimate.

\section{B. The Adaptive Interference Canceller}

In practical systems, however, the rejection of the desired signal in $\mathrm{v}(\mathrm{n})$ is limited by matching, just like the HR in the analog down-mixer. Fortunately, the AIC technique does not require perfect rejection of the desired signal to give good results. Consider a third harmonic interferer and a desired signal that are equally strong after passing through the analog HR down-mixing stage. Given a realistic (matching limited) desired signal rejection of $40 \mathrm{~dB}$ during the interference estimate generation by way of (6), the SIR of the estimate, $\mathrm{SIR}_{v(n)}$, is $-40 \mathrm{~dB}$. Using (5), the theoretical SIR after the AIC, $\operatorname{SIR}_{e(n)}$, is $40 \mathrm{~dB}$. Then the total harmonic rejection is $40 \mathrm{~dB}$ plus the rejection obtained by the analog first stage (typically in the range of 30 to $40 \mathrm{~dB})$.

Given the above, it should be clear that the additional harmonic rejection provided by the AIC is dependent on the SIR of the baseband signals $I Q_{1}$ and $I Q_{2}$, which is equal to the signal-to-harmonic ratios of the RF antenna signal minus the HR of the analog front-end.

Interestingly, the performance of the AIC shows a favorable trend with respect to the interference power: if the interference power increases, the quality (1/SIR) of the interference estimate increases, which leads to an increased SIR at the output of the canceller. In practice, the benefit of this trend is limited by the nonlinearity of the front-end, including the A/D converters.

Consider again the block diagram of the digital HR stage in Fig. 9. The interference estimate, v(n), and its complex conjugate, ${ }^{6} v^{*}(n)$, are equalized via multiplying by $w_{1}^{*}$ and $w_{2}^{*}$, re-

\footnotetext{
${ }^{6}$ The complex conjugate is needed to remove the I/Q imbalance image [27]
} of the harmonic image in addition to the harmonic image itself. spectively. The equalized signals are subtracted from the received signal, $r(n)$, which removes the interference and produces the output signal, e(n). The filter weights, $w_{1}^{*}$ and $w_{2}^{*}$, are adapted with every new output value of e(n) by means of the LMS update rule [25]:

$$
\begin{aligned}
& w_{1}(n+1)=w_{1}(n)+\mu \cdot v(n) \cdot e^{*}(n) \\
& w_{2}(n+1)=w_{2}(n)+\mu \cdot v^{*}(n) \cdot e^{*}(n)
\end{aligned}
$$

where $\mu$ is the power-normalized step-size, normalized to the power of the interference estimate $\mathrm{v}(\mathrm{n})$, i.e., $P_{v}$ :

$$
\mu=\frac{1 \cdot 10^{-4}}{P_{v}}
$$

and the canceller output, e(n), is calculated from the received signal, $r(n)$, by

$$
e(n)=r(n)-w_{1}^{*}(n) \cdot v(n)-w_{2}^{*}(n) \cdot v^{*}(n)
$$

as shown in Fig. 9.

The LMS update rule as in (7) is an iterative process that aims to minimize the cross-correlation between the output of the canceller, e(n), and the interference estimate, $v(n)$. Cross-correlation is a measure of similarity, thus, minimizing it results in the output of the canceller being as dissimilar to the interference estimate as possible: the interference at the output, e(n), is reduced.

The step-size parameter $\mu$ in (8) is chosen somewhat arbitrarily. Generally speaking, choosing $\mu$ too small results in slow convergence and choosing it too big increases the (time-varying) error of the filter weights [25], which reduces the harmonic rejection.

The optimum equalizer coefficients, $w_{1}$ and $w_{2}$, for cancelling the third harmonic image may differ from the optimum coefficients for cancelling the fifth harmonic image, owing to different phase and amplitude mismatches for each image. The dominating interference largely determines the cross-correlation. Therefore, the dominating harmonic image will be cancelled by the AIC stage. Note that the preceding analog HR down-mixer stage rejects both images.

The optimum coefficients are independent of the RF signal modulation scheme, owing to the fact that the amplitude and phase differences between $\mathrm{r}(\mathrm{n})$ and $\mathrm{v}(\mathrm{n})$ are independent of the actual RF signals. Thus, once the filter coefficients to cancel a specific harmonic image have been found (by application of the iterative LMS algorithm), they remain valid until the mismatch introduced by the front-end changes, for instance, when making large changes in the LO frequency.

\section{IMPLEMENTATION OF THE ANALOG FRONT-END}

A SDR receiver chip has been implemented in $65 \mathrm{~nm}$ CMOS to verify the three concepts proposed in previous sections. The digital AIC algorithm is realized in software and will be discussed later. The block diagram of the chip has been shown in Fig. 4. The signal path consists of LNTAs, passive mixers, and two-stage TIAs with second-stage HR-weighting via a resistor network (R-net). The first voltage gain should be at baseband after LPF for good OB linearity, as discussed in Section II, and the realization of two-stage polyphase HR has been described in 


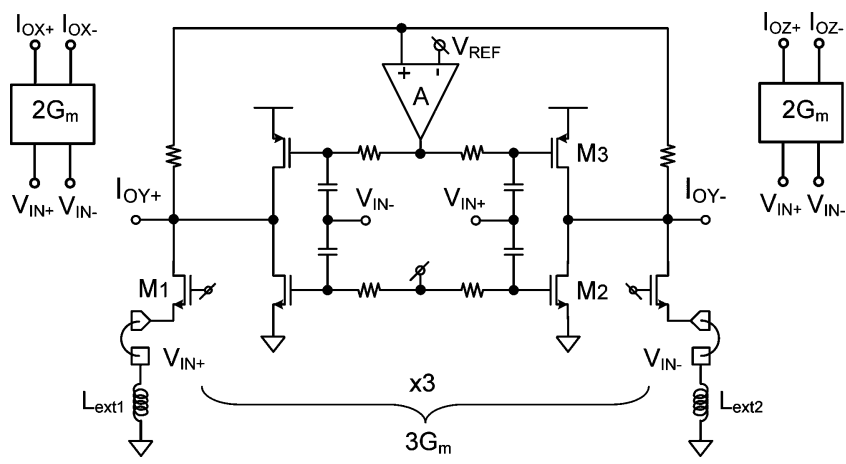

Fig. 10. Low-noise transconductance amplifiers (LNTA) implementing $3 G_{m}$ (shown on transistor level) and two blocks of $2 G_{m}$ (identical schematic).

Section III. The 8-phase LO is derived via a divide-by-8 from an off-chip signal CLK, i.e., the master clock. The receiver can be reconfigured to deliver either 8-phase outputs from TIA1 or I/Q outputs from TIA2. The 8-phase outputs interface to off-chip ADCs for digitally enhanced HR measurements while the TIA2 stage is switched off. To better understand the implementation, a more detailed description for some key blocks follows.

\section{A. Linear LNTA}

Fig. 10 shows the schematic of a pseudo-differential unit-LNTA, of which there are 7 units in parallel to form three LNTAs with 2:3:2 ratio, sharing the same external (large-value) inductor to GND for DC bias. The common-gate (CG) transistor M1 provides input matching while the input is also connected to the AC-coupled inverter consisting of common-source (CS) transistors M2 and M3. For each single-ended half, all 7 unit-LNTAs together deliver an impedance matching with the source impedance $R_{S}=50 \Omega$ and a total transconductance $g_{m, t o t}=100 \mathrm{mS}\left(g_{m, C G}=20 \mathrm{mS}, g_{m, C S}=80 \mathrm{mS}\right)$. A common-mode feedback (CMFB) loop using high-ohmic resistors and an amplifier "A" controls the PMOS transistors and ensures all three LNTA outputs are biased around $V_{\mathrm{REF}}=600 \mathrm{mV}$. In total the three differential LNTAs draw $14 \mathrm{~mA}$ from a $1.2 \mathrm{~V}$ supply.

The noise behavior of the LNTA can be understood by studying a single-ended half, which consists of a CG transistor M1 and two CS transistors M2/M3, sharing the same input $V_{\mathrm{in}+}$. Considering the LNTA output noise in the current domain, the noise factor can be written as

$F=1+\frac{k T \gamma g_{m, C G} \cdot\left(1-R_{S} \cdot g_{m, C S}\right)^{2}}{k T R_{S} \cdot g_{m, t o t}^{2}}+\frac{4 k T \gamma g_{m, C S}}{k T R_{S} \cdot g_{m, t o t}^{2}}$.

The second term considers the partial noise cancelling of the CG transistor noise [28] and the third term considers the noise from the CS transistors. If take $R_{S}=50 \Omega, g_{m, C G}=20 \mathrm{mS}$, $g_{m C S}=80 \mathrm{mS}$, and $g_{m, t o t}=100 \mathrm{mS}$ into $(10)$, we get $F=$ $1+9 \gamma / 25+16 \gamma / 25=1+\gamma$. If $\gamma$ is in the range of $2 / 3$ to 1 , the noise figure (NF) would be 2.2 to $3 \mathrm{~dB}$.

For wideband operation, minimum-length transistors are used to achieve $S_{11}<-10 \mathrm{~dB}$ to more than $6 \mathrm{GHz} \mathrm{RF}$ (simulation). Since the input impedance of a CG transistor depends on its drain impedance [29], a wideband low impedance at its output,

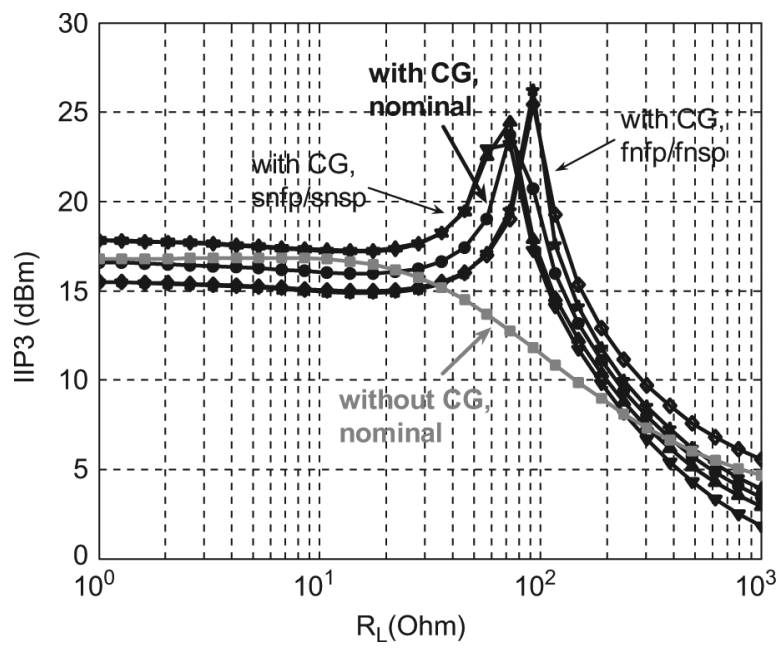

Fig. 11. Simulated LNTA IIP3 versus load impedance $\left(R_{L}\right.$ for each of the three LNTAs) at different process corners (sn: slow-NMOS, sp: slow-PMOS, fn: fast-NMOS, fp: fast-PMOS).

i.e., node B in Fig. 2, is desired for wideband input matching. This fits well to what is desired for linearity as discussed in Section II.

Since the LPF improves the OB linearity of I-V conversion (Fig. 1), the V-I linearity sets the ultimate limit of OB linearity. To obtain a good V-I linearity, high $\left(V_{G S}-V_{T H}\right)$ and high $V_{D S}$ is desired. In our $V D D=1.2 \mathrm{~V}$ design, $\left(V_{G S}-V_{T H}\right)$ is larger than $250 \mathrm{mV}$ and $V_{D S}$ is $600 \mathrm{mV}$. Fig. 11 shows IIP3 simulation results (considering process spread), where each of the three LNTAs is loaded by a pair of resistors $R_{L}$ to model the input impedance of the next stage mixers. To average out the effect of different LNTA transconductance (2:3:2), the simulation is carried out with all three LNTAs combined together as well as their individual $R_{L}$. The two input tones are at $801 \mathrm{MHz}$ and $802 \mathrm{MHz}$. Simulations predict an IIP3 of more than $+15 \mathrm{dBm}$ if $R_{L}<100 \Omega$ and only $\pm 1 \mathrm{~dB}$ variation over different process corners, indicating that high LNTA linearity robust to process spread is possible if we keep voltage gain low (small $R_{L}$ ).

Actually, it turns out that the addition of the CG-stage in parallel to the inverters has a nonlinearity cancellation effect that improves IIP 3 for $R_{L}$ between about $20 \Omega$ and $200 \Omega$, which determines the $V_{d s}$-related distortion terms (for the case without a CG-stage, see the grey curve in Fig. 11). Simulation and analysis indicate that it is mainly the pre-distortion at the inverter inputs introduced by the CG-stage via its source current, to cancel the distortion generated by inverter itself. Nevertheless, since we are interested in using a low $R_{L}$ value, produced by mixer switches, to deliver signal current into the TIA stage, here we do not discuss this effect further.

A differential LNTA requires an off-chip balun if a singleended antenna or RF filter is used. Compared to an LNTA with single-ended input, although the differential one may double the power consumption [30], it can render better IIP2. Besides, the input voltage swing on each of the differential inputs is lowered by $3 \mathrm{~dB}$, which improves LNTA IIP 3 and $P_{1 \mathrm{~dB}}$ by $3 \mathrm{~dB}$.

Using the same setup as for Fig. 11, simulations with an ideal balun and $R_{L}=50 \Omega$ (the designed input impedance of the mixer) show input-referred $P_{1 \mathrm{~dB}}=+4 \mathrm{dBm}, \mathrm{NF}<3 \mathrm{~dB}$ (only 
including noise from LNTA), and voltage gain $=-1 \mathrm{~dB}$ for each single-ended output (low gain as desired for good linearity) with $-3 \mathrm{~dB}$ bandwidth $>7 \mathrm{GHz}$. This wide RF bandwidth benefits from the low impedance (real part) at the output of LNTA, which means the dominant pole is located at a very high frequency given a certain capacitance.

\section{B. Passive Mixer}

Each of the three LNTAs with 2:3:2 ratio connects to 8 passive current-commutating mixers driven by 8 -phase LO, as shown in Fig. 4. The mixers are DC-coupled to the LNTAs for wider bandwidth compared to AC-coupled, which introduces parasitic capacitance. Each mixer receives 3 differential inputs from LNTAs and together they deliver 4 differential outputs to TIA1, i.e., 8-phase signals with $45^{\circ}$ interval.

The passive mixer simply consists of NMOS switches, with bulk tied to source. The gate of the mixer switch is AC-coupled to a clock driver and biased so that the maximum $V_{G S}=\mathrm{VDD}$. The mixer switch-on resistance $R_{\text {mixer }}$ is in the order of $50 \Omega$ and all mixer switches have the same dimension for good phase accuracy. Besides, the same $R_{\text {mixer }}$ and different LNTA output impedance (3:2:3) also introduce a current division effect which brings the actual first-stage weighting ratio different from $2: 3: 2$ but closer to the ideal $1: \sqrt{2}: 1$ ratio, good for the overall amplitude accuracy.

For good NF, we need to minimize clock overlap to avoid a low-ohmic path between TIA inputs that will amplify TIA noise [22]. For the case with 8 TIA inputs this leads to a maximum LO duty cycle of $1 / 8$. Both sides of the mixer, i.e., the output of LNTA and the input of TIA, are biased at the same DC level (around half VDD) ensuring that little DC current flows for a low $1 / \mathrm{f}$ noise from the mixer switches.

\section{Accurate Multiphase Clock}

Since the amplitude accuracy can be ensured by the two-stage polyphase HR, the phase inaccuracy is likely to dominate. Based on the Appendix, if the LO duty cycle is "d", the resulting third $\operatorname{HR}(1 \sigma)$ is

$$
H R 3=10 \log \left(\frac{\sin ^{2}(\pi \cdot d)}{\sin ^{2}(3 \pi \cdot d)} \cdot\left[\left(\frac{\sigma_{A}}{12}\right)^{2}+\left(\frac{\sigma_{\varphi}}{4}\right)^{2}\right]^{-1}\right)
$$

where $\sigma_{A}$ and $\sigma_{\varphi}$ are the standard deviation in the amplitude and phase respectively. For $d=1 / 8$ and negligible amplitude error $\left(\sigma_{A} \rightarrow 0\right)$ due to the two-stage technique as in (4), to reach $60 \mathrm{~dB} \operatorname{HR}(3 \sigma)$, the required phase error is $\sigma_{\varphi}=0.03^{\circ}$.

To build a multiphase clock generator with low phase mismatch, two design principles are applied: 1) to use a common master clock to derive all phases; 2) to minimize the path from the common master clock to the mixer switches therefore to minimize mismatch accumulation.

Fig. 12 shows a divide-by-8 ring counter using eight dynamic transmission-gate (TG) flip-flops (FF). The same master clock (CLK), with 8 times the LO frequency, drives all FFs. Only one inverter (INV2) is used as a buffer to minimize the path from CLK to mixer.

A preset data pattern is required to deliver the wanted $1 / 8$ duty cycle. Each LO phase controls six mixer switches connecting to

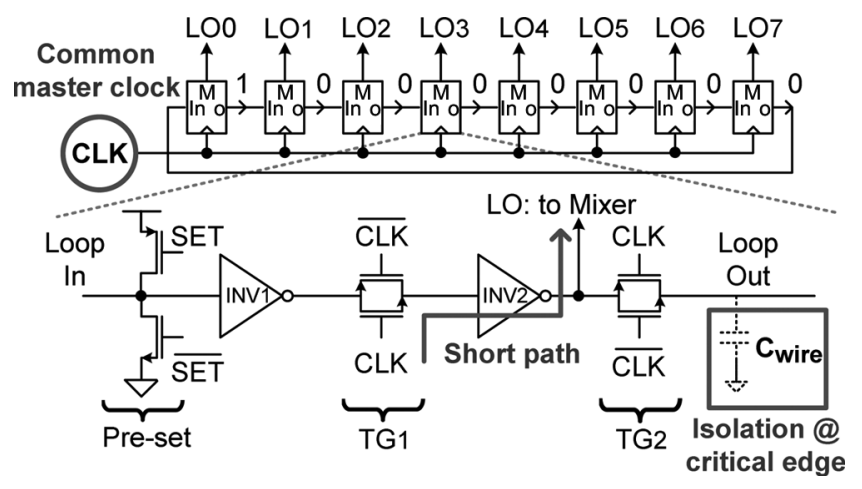

Fig. 12. An 8-phase clock generator with low phase mismatch (with one cell shown on transistor level).

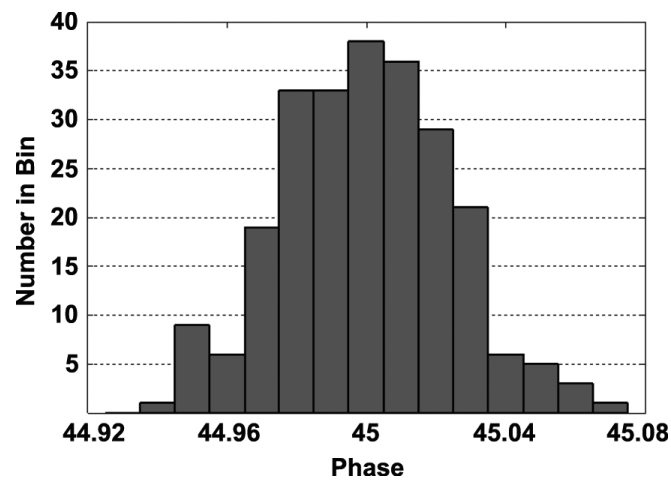

Fig. 13. Histogram of the simulated phase difference between two adjacent LO outputs (240 Monte Carlo results).

differential outputs of three LNTAs. The gates of all six switches are connected together and driven by the same buffer, i.e., INV2, to minimize buffer mismatch.

In a ring counter, all flip-flops "see" the same environment. However, a loop is not convenient in layout and it may need different wiring lengths between each two flip-flops, degrading phase accuracy. A careful layout strategy is adopted to minimize the wiring differences. Moreover, when the critical LO edges occur, the largest part of the wiring is isolated from the output of INV2 via TG2, decreasing rise and fall times and reducing the effect of wiring mismatch.

The phase error reported in [17] is found to be too pessimistic due to an incorrect simulation test-bench. Fig. 13 presents the simulated phase deviation from $45^{\circ}$ between two adjacent $0.8 \mathrm{GHz}$ LO phases due to mismatch, including the contribution from mixer switches. The histogram shows a maximum phase error of only $0.07^{\circ}$ and it yields $\sigma=0.024^{\circ}$, i.e., $0.08 \mathrm{ps}$ for $0.8 \mathrm{GHz}$. This clock performance is hence compatible with $H R>60 \mathrm{~dB}(3 \sigma)$.

The master clock CLK comes from an off-chip generator followed by a pair of inverters as on-chip buffer. Simulation shows, at $0.8 \mathrm{GHz} \mathrm{LO}$, the power consumption of the divider is $5.4 \mathrm{~mA}$ at $1.2 \mathrm{~V}$ supply and the input buffers consume $8.9 \mathrm{~mA}$ driven by $6.4 \mathrm{GHz}$ differential input clock.

In simulation, the divide-by- 8 can work up to $1.25 \mathrm{GHz} \mathrm{LO}$ $(10 \mathrm{GHz} \mathrm{CLK})$ in nominal case although it can vary with process corners. The up-side LO frequency is mainly limited by the large 
division ratio, i.e., 8. If a higher $\mathrm{LO}$ frequency is wanted, a divide-by-2 may be used to generate 4-phase (quadrature) LO instead of the divide-by- 8 , and then the receiver in Fig. 4 can be reconfigured to a quadrature wideband receiver without HR [21], when harmonic mixing is less to be a problem at higher bands.

\section{High-Swing TIA and Baseband R-net}

Since the voltage gain occurs at the outputs of the TIA1 stage where interference is only partly suppressed, we choose an OTA topology [21] being able to handle large voltage swing, which helps tolerate large blockers. It is a two-stage class-AB-output OTA based on [31]. The input pair uses NMOS transistors in weak inversion for high $g_{m} / I_{\mathrm{DC}}$ and a big size leading to low $1 / \mathrm{f}$ noise. For the OTA second stage, a class-AB push-pull output stage is used, which can handle more than $2 \mathrm{~V}$ peak-to-peak differential output voltage swing. Each OTA draws $3 \mathrm{~mA}$ from $1.2 \mathrm{~V}$ supply.

A parallel $R C$ feedback network implements a simple first order LPF to perform blocker filtering (Fig. 4). Each TIA stage has a LPF $-3 \mathrm{~dB}$ bandwidth of $20 \mathrm{MHz}$ and together they determine the receiver IF bandwidth of $12 \mathrm{MHz}$, which may accommodate most mobile communication standards. The virtual-ground impedance of the TIAs is about $4 \Omega$ around DC and peaks to $60 \Omega$ around $700 \mathrm{MHz}$. The simulated gain after the TIA1 stage is $27 \mathrm{~dB}$ and after the TIA2 stage $34 \mathrm{~dB}$.

The resistor network (R-net) provides the second-stage weighting for HR. It also converts 8-phase outputs of the TIA1 stage into quadrature inputs of the TIA2 stage. To form a 5:7:5 amplitude ratio, 19 unit-resistors form a resistance ratio of 7:5:7 in three paths. Harmonic rejection at baseband (via R-net) can also reduce errors due to parasitic capacitance compared to at high frequency.

\section{IMPLEMENTATION OF THE DigITAL BACK-END}

The analog front-end used in the digitally enhanced HR architecture consists of the first stage HR mixer driven by the multi-phase clock generator of the two-stage analog HR architecture. The reader is referred to the previous section for the implementation details of the analog circuits.

The four fully differential baseband outputs provided by the TIA1 stage (Fig. 4) are converted into the digital domain using a commercial A/D board comprising four 14-bit ADCs (Fig. 9). Unfortunately, the input range of the used A/D board was more than 15 times greater than the output swing provided by the front-end, resulting in less than 10 effective bits.

The baseband processing, including the interference estimate generation and the adaptive interference canceller were implemented in software on a PC and use floating-point arithmetic. To allow real-time processing, a sampling rate of $4 \mathrm{MS} / \mathrm{s}$ was chosen. This gives $2 \mathrm{MHz}$ bandwidth for each analog baseband signal and $4 \mathrm{MHz}$ bandwidth in the digital domain using quadrature signals. Fig. 9 gives a system-level overview of the setup.

The interference estimate generation is implemented using two real adders and the phase shifter, shown in Fig. 14. This reduced-complexity shifter exploits the fact that the cosine and sine of a $45^{\circ}$ angle are of equal magnitude. Thus, it needs two real multipliers (instead of four) and two real adders. Thus, the

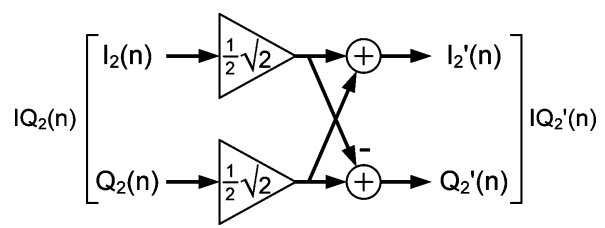

Fig. 14. Reduced complexity $-45^{\circ}$ phase shifter.

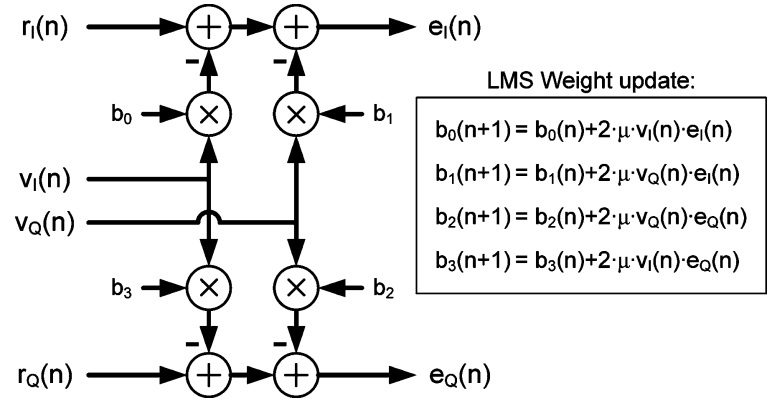

Fig. 15. Reduced complexity interference canceller.

total complexity of the interference estimate generation is two real multipliers and four real adders.

The complexity of the canceller indicated by (9) can be reduced from eight multipliers and eight adders to four multipliers and four adders, by applying the following substitutions:

$$
\begin{array}{ll}
b_{0}=w_{1, I}+w_{2, I} & b_{1}=w_{1, Q}-w_{2, Q} \\
b_{2}=w_{1, I}-w_{2, I} & b_{3}=-w_{1, Q}-w_{2, Q}
\end{array}
$$

where the filter coefficients, $w_{1}$ and $w_{2}$, are split in their real and imaginary parts, $w_{1, I}, w_{1, Q}$, etc. The resulting canceller and the new LMS update rules are shown in Fig. 15. If the step-size $\mu$ is rounded to the nearest power of 2 , four multipliers in the "LMS Weight update" become a shift operation. As a result, the update mechanism only needs four multipliers and four adders. Then, the total arithmetic complexity of the digital HR stage is 10 multiplications and 12 additions per sample.

While the digital algorithm was implemented only in software, a fixed-point VHDL version was synthesized using a $65 \mathrm{~nm}$ CMOS standard cell library. The tools reported a dynamic power of less than $10 \mathrm{~mW}$ at $100 \mathrm{MS} / \mathrm{s}$ and $1.2 \mathrm{~V}$ supply voltage.

\section{EXPERIMENTAL RESULTS}

The circuit shown in Fig. 4 is fabricated in $65 \mathrm{~nm}$ CMOS and the micrograph is shown in Fig. 16. The total area, excluding bond-pads, is about $1 \mathrm{~mm}^{2}$. Capacitors $\left(C_{\mathrm{FB}}\right.$ and $C_{\mathrm{VG}}$ in Fig. 2) take a large portion of area in the TIA, and also the OTA input pair is big to achieve a low $1 / \mathrm{f}$ noise corner. With $1.2 \mathrm{~V}$ supply, the analog power consumption is $33 \mathrm{~mA}$ (LNTA: $14 \mathrm{~mA}$, TIA1stage: $12.8 \mathrm{~mA}$, TIA2-stage: $6.4 \mathrm{~mA}$ ) while the clock power consumption is $8 \mathrm{~mA}$ at $0.4 \mathrm{GHz} \mathrm{LO}$ and $17 \mathrm{~mA}$ at $0.9 \mathrm{GHz}$ LO, including the clock input buffers.

The chip is packaged in a 32-pin heat-sink very-thin quad flat-pack no-leads (HVQFN) package. To prove the receiver is robust to OBI, all measurements are performed on PCB without any external filter. Two SMD inductors are mounted on the PCB to bias the LNTA (Fig. 10). Both the receiver inputs and clock 


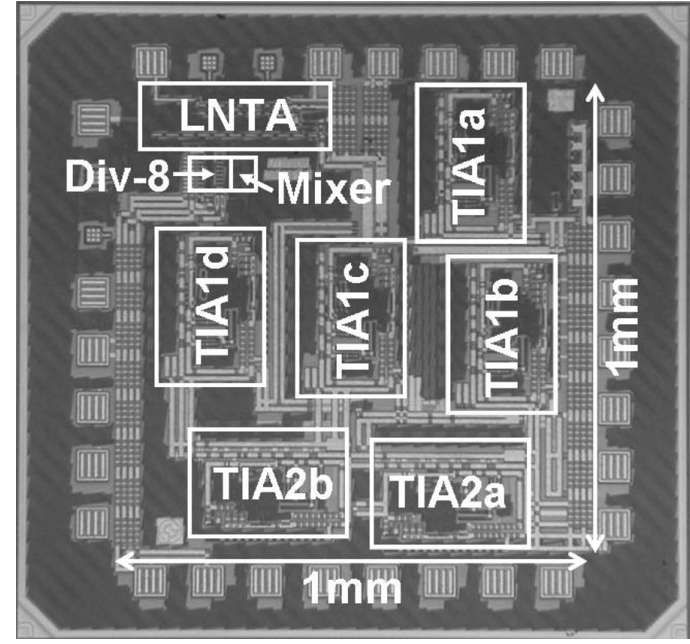

Fig. 16. $65 \mathrm{~nm}$ CMOS chip micrograph indicating some functional blocks.

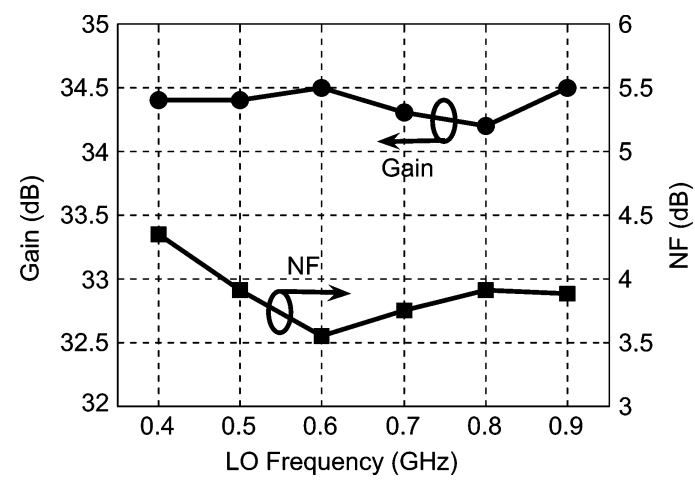

Fig. 17. Measured voltage gain and DSB NF of the two-stage receiver as a function of the $\mathrm{LO}$ frequency.

inputs are differential and wideband hybrids (balun) were used to interface to single-ended $50 \Omega$ measurement equipments. The IF-output voltages are sensed by a differential active probe that performs differential to single-ended conversion and impedance conversion to $50 \Omega$. The characteristics of all components and cables for testing are de-embedded from the results.

The divide-by- 8 works up to $0.9 \mathrm{GHz} \mathrm{LO}$, and the measured $S_{11}$ is lower than $-10 \mathrm{~dB}$ up to $5.5 \mathrm{GHz}$. This means the HR measurement is valid for $0.9 \mathrm{GHz} \mathrm{LO}$ up to its sixth harmonic. The measured IF bandwidth is $12 \mathrm{MHz}$ and the baseband $1 / \mathrm{f}$ noise corner is $30 \mathrm{kHz}$ thanks to the passive mixer with little DC current and the OTA with a large-sized input pair.

\section{A. Gain, NF, RF Bandwidth, and In-Band IIP2/IIP3}

Fig. 17 shows the measured voltage gain and DSB NF over an LO frequency of 0.4 to $0.9 \mathrm{GHz}$. The voltage conversion gain, measured for an IF of $1 \mathrm{MHz}$ from the input of the balun to the differential outputs of receiver, is above $34 \mathrm{~dB}$ over the whole band and is quite flat $( \pm 0.2 \mathrm{~dB}$ variation), indicating a much wider RF bandwidth. The NF is measured for an IF of $10 \mathrm{MHz}$ since the available NF analyzer (Agilent N8973 A) starts from that frequency. The DSB NF is below $4 \mathrm{~dB}$ except for $0.4 \mathrm{GHz}$ where $1 / \mathrm{f}$ noise from the LNTA starts to dominate.

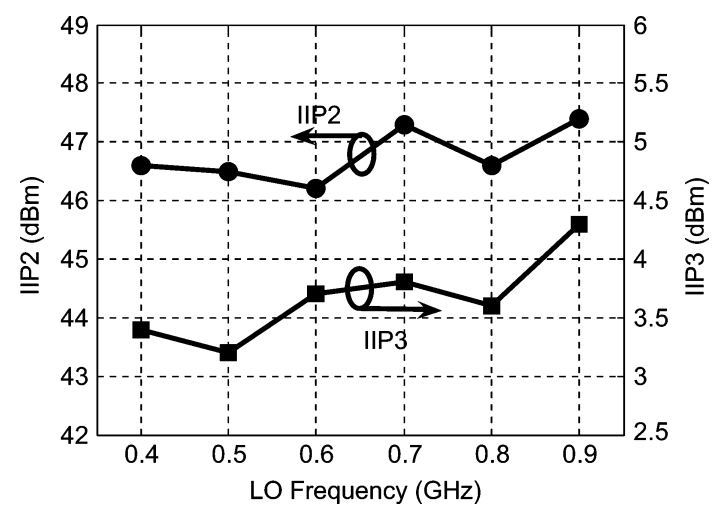

Fig. 18. Measured in-band IIP2 and IIP3 versus LO frequency.

The divide-by- 8 limits the $\mathrm{LO}$ frequency range up to $0.9 \mathrm{GHz}$ (master clock@ $7.2 \mathrm{GHz}$ ), but the signal-path $-3 \mathrm{~dB}$ RF bandwidth is much wider, up to $6 \mathrm{GHz}$. To verify it, we conducted a gain measurement for the seventh harmonic, i.e., the first noncanceled high-order harmonic. Ideally, using 1/8 duty-cycle LO, the strength of the seventh harmonic should be $1 / 7$ of the fundamental harmonic, so we expect the seventh harmonic should ideally have a gain that is $16.9 \mathrm{~dB}(1 / 7)$ lower from $34 \mathrm{~dB}$, i.e., $17.1 \mathrm{~dB}$. Indeed, the gain drops from $17 \mathrm{~dB}$ at $0.7 \mathrm{GHz} \mathrm{RF}$ to $14.3 \mathrm{~dB}$ at $6 \mathrm{GHz} \mathrm{RF}$ (LO: 0.1 to $0.85 \mathrm{GHz}$ ), which means the OBI will only be attenuated a little by the frequency roll-off at $\mathrm{RF}$. It also indicates that the receiver can be readily expanded to cover higher bands by extending the $\mathrm{LO}$ range as discussed in Section V-C.

Fig. 18 shows the measured in-band (IB) IIP2 and IIP3 over LO frequency, with two tones close to the $\mathrm{LO}$ frequency so that they are not affected by IF filtering (IIP2: $f_{\mathrm{LO}}+3 \mathrm{MHz}$ and $f_{\mathrm{LO}}+6.01 \mathrm{MHz} ;$ IIP3: $f_{\mathrm{LO}}+3 \mathrm{MHz}$ and $f_{\mathrm{LO}}+3.01 \mathrm{MHz}$ ). After downconversion, the IM2 component at $3.01 \mathrm{MHz}$ and the IM3 component at $2.99 \mathrm{MHz}$ are measured. The IB IIP3 is around $+3.5 \mathrm{dBm}$, which is good given the high gain of $34 \mathrm{~dB}$, thanks to only voltage gain at baseband with negative feedback. The IB IIP2 is above $+46 \mathrm{dBm}$.

\section{B. Out-of-Band IIP2/IIP3}

We also measured the out-of-band (OB) IIP2 and IIP3. Due to the LPF behavior, the measured OB linearity depends on the distance from $f_{\mathrm{LO}}$ to the two RF tones used. For sufficient distance, the LPF will suppress the downconverted two-tone interference so the OB nonlinearity is mainly contributed by the V-I of the LNTA.

The OB IIP3 is tested via two tones at $1.61 \mathrm{GHz}$ and $2.40 \mathrm{GHz}$ with an LO at $819 \mathrm{MHz}$, so that the IM3 is at $820 \mathrm{MHz}$ RF and $1 \mathrm{MHz}$ IF. The results of both IB $(0.8 \mathrm{GHz} \mathrm{LO})$ and OB IIP3 are shown in Fig. 19. Without fine tuning, the measured OB IIP3 is $+16 \mathrm{dBm}$, which agrees with the simulated results in Fig. 11. Compared to the IB IIP3 of $+3.5 \mathrm{dBm}$, the OB IIP3 is dramatically improved because the TIA was dominating the IB IIP3, due to the high voltage gain at the output. As shown in the figure, the range for which IM3 follows the extrapolation line is also improved by almost $20 \mathrm{~dB}$ (upper limit of $-30 \mathrm{dBm}$ 


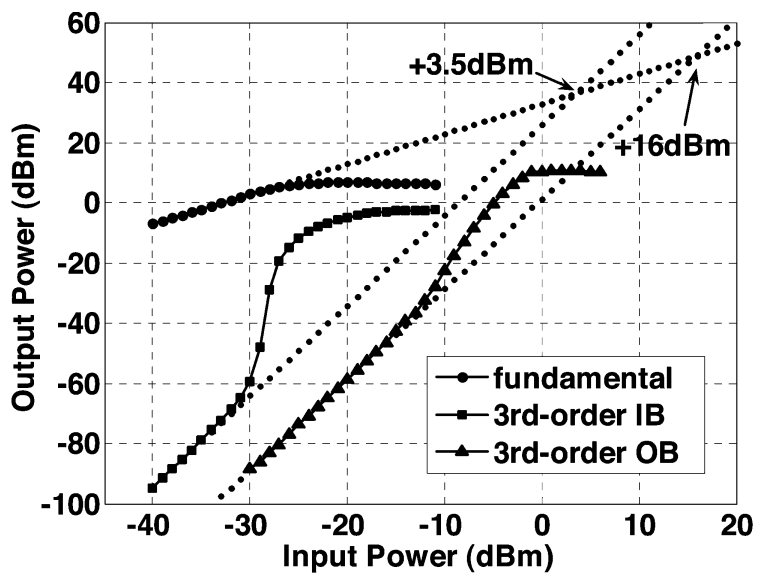

Fig. 19. Measured in-band (IB) and out-of-band (OB) IIP3 for $800 \mathrm{MHz}$ LO demonstrating OB linearity improvement.

for $\mathrm{IB}$ versus $-10 \mathrm{dBm}$ for $\mathrm{OB})$. This is crucial to tolerate large $\mathrm{OB}$ interference.

The OB IIP2 is $+56 \mathrm{dBm}$, tested via two tones at $1.80 \mathrm{GHz}$ and $2.40 \mathrm{GHz}$ while $\mathrm{LO}$ at $601 \mathrm{MHz}$, so that the IM2 is at $600 \mathrm{MHz}$ RF and $1 \mathrm{MHz}$ IF.

\section{C. $1 \mathrm{~dB}$ Compression Point and Blocker Filtering}

To quantify the effect of the blocker filtering, we measured the $1 \mathrm{~dB}$ compression point $\left(P_{1 \mathrm{~dB}}\right)$ and the $1 \mathrm{~dB}$ desensitization point $\left(B_{1 \mathrm{~dB}}\right)$, both input-referred.

First we measured the $P_{1 \mathrm{~dB}}$ without applying any blockers, which is $-22 \mathrm{dBm}$. The result is reasonable since $-22 \mathrm{dBm}$ input power plus $34 \mathrm{~dB}$ gain is equal to $12 \mathrm{dBm}$ output power (referring to $50 \Omega$ ), differentially. The single-ended voltage swing is about $1.27 \mathrm{~V}$ peak to peak, just exceeding the $1.2 \mathrm{~V}$ supply. This means the limitation is at the receiver output and the $P_{1 \mathrm{~dB}}$ can be improved by automatic gain control (AGC).

A more serious problem is to receive a weak signal at the same time with a strong interferer: a so-called blocker test. In this situation AGC does not help since the maximum gain is required to maintain sensitivity. The measurement was carried out with the $\mathrm{LO}$ at $400 \mathrm{MHz}$ and the desired RF signal at $401 \mathrm{MHz}$ with $-50 \mathrm{dBm}$ input power. The blocker frequency is varied from $402 \mathrm{MHz}$ to $4.002 \mathrm{GHz}$. Fig. 20 shows $B_{1 \mathrm{~dB}}$ versus the blocker frequency. As predicted by (1) and (2), we see two effects in the figure: 1) the tolerable blocker power depends on the frequency distance between the $\mathrm{LO}$ and the blocker, due to the LPF behavior; ${ }^{7}$ 2) HR also plays a role in blocker filtering, as two dips occur around seventh and ninth harmonic of the LO frequency, both of which are not rejected well by the 8-phase HR. From the figure, we can observe that $B_{1 \mathrm{~dB}}$ is better than $P_{1 \mathrm{~dB}}(-22 \mathrm{dBm})$ except very close-by blockers $(402 \mathrm{MHz})$ and the maximum $B_{1 \mathrm{~dB}}$ is more than $0 \mathrm{dBm}$, showing the blocker filtering is indeed effective.

\section{Two-Stage Polyphase HR}

We will verify the analog two-stage polyphase HR here and the digitally enhanced HR in Section VII-E. These two alternative approaches will be compared in Section VII-F.

\footnotetext{
${ }^{7}$ The actual behavior of the LPF is more complicated than (2), since our baseband filter is cascaded in two stages, which does not follow a simple first order or second order filtering behavior.
}

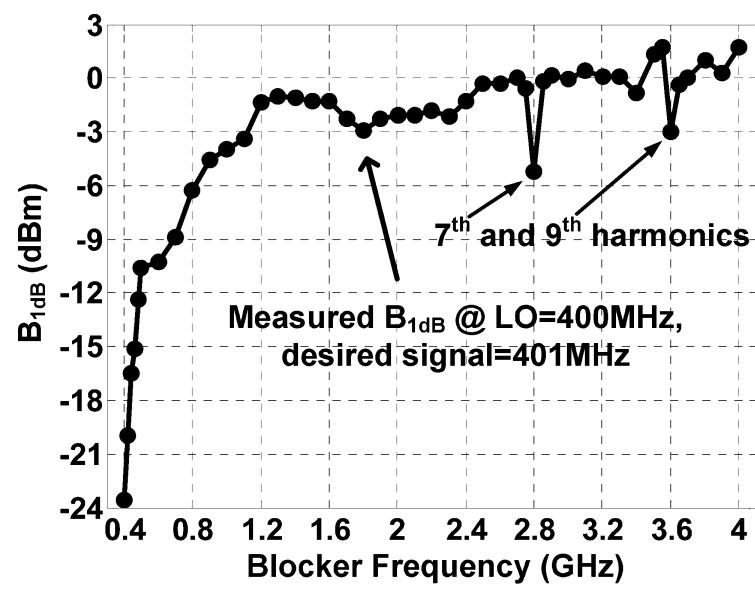

Fig. 20. Measured input-referred $1 \mathrm{~dB}$ desensitization point $\left(B_{1 \mathrm{~dB}}\right)$ versus blocker frequency.

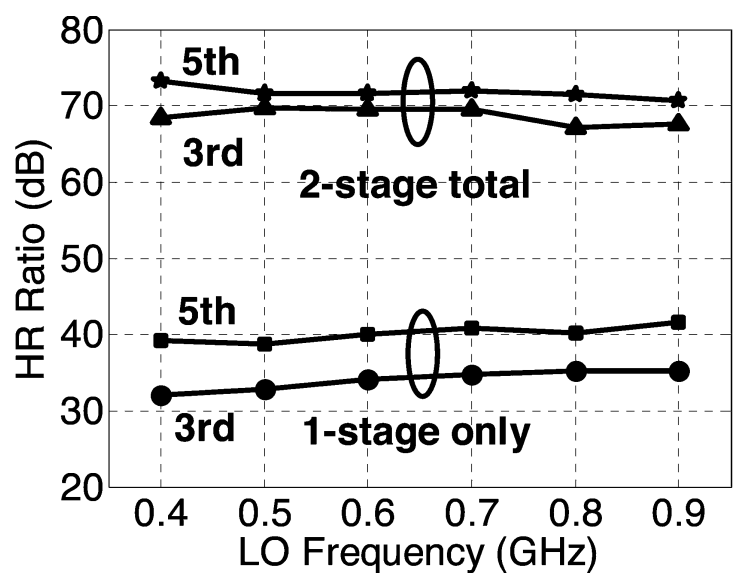

Fig. 21. Measured HR ratio versus LO frequency: Comparison between HR with only one-stage and total two-stage (two-stage polyphase HR).

First we look at the two-stage polyphase HR. The HR ratio can be measured by comparing the gain difference between the desired signal and the harmonic image. At the receiver input, the desired signal power was $-50 \mathrm{dBm}$ while the harmonic image power was $-30 \mathrm{dBm}$.

Fig. 21 shows, for one chip, the HR of one-stage, at the outputs of the TIA1, and the total two-stage HR, at the outputs of the TIA2, versus LO frequency. The HR of one-stage is between 30 and $40 \mathrm{~dB}$ and the HR of two-stage is around $70 \mathrm{~dB}$, representing a $30 \mathrm{~dB}$ improvement for both third and fifth HR thanks to the two-stage polyphase HR technique. Generally, the HR improvement from one-stage to two-stage is in the range of 20 to $40 \mathrm{~dB}$ as observed from multiple chips. The large improvement also shows that it is the amplitude error dominating the first-stage HR.

To identify the effect of mismatch, we measured the HR of two-stage for 40 chips at $0.8 \mathrm{GHz} \mathrm{LO}$, as shown in Fig. 22. The minimum third order $\mathrm{HR}$ is $60 \mathrm{~dB}$ and the minimum fifth order HR is $64 \mathrm{~dB}$. The second, fourth, and sixth HR is also measured, over 20 chips. The minimum second order HR is $62 \mathrm{~dB}$, while the minimum fourth and sixth order HR are both $67 \mathrm{~dB}$. These results are achieved without calibration, trimming, or RF filtering. 


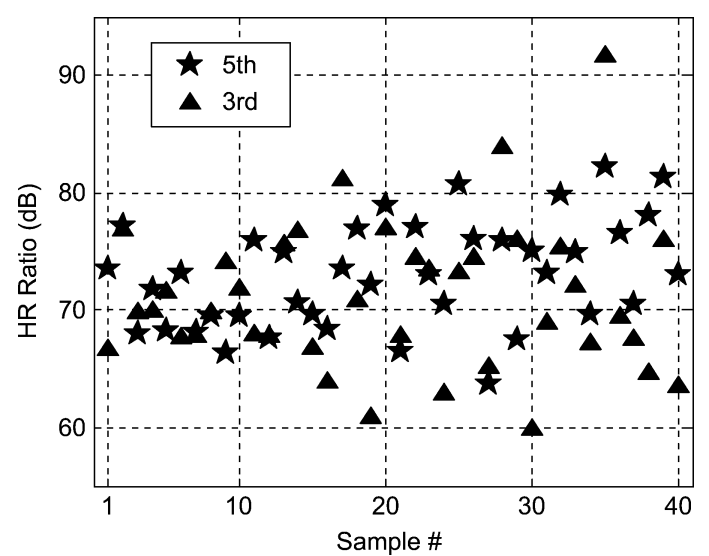

Fig. 22. Measured HR ratio of 40 randomly selected chips at $800 \mathrm{MHz} \mathrm{LO}$ (two-stage polyphase HR).

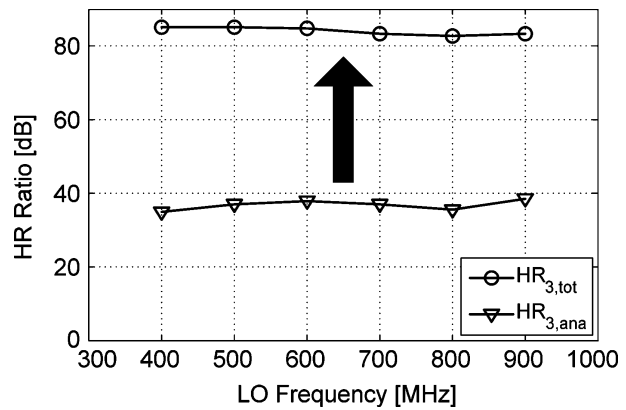

Fig. 23. The measured third order HR of the analog stage and the combined stages versus the $\mathrm{LO}$ frequency. Desired: $-66.1 \mathrm{dBm}$ RF power, third harmonic image: $-20.1 \mathrm{dBm}$ RF power (digitally enhanced HR).

Since the signal-path $-3 \mathrm{dBm}$ RF bandwidth has been characterized to be up to $6 \mathrm{GHz}$, the contribution of the frequency roll-off to the HR result should be small. According to (11), the simulated phase error $\sigma=0.024^{\circ}$ means a minimum HR $(3 \sigma)$ of $62 \mathrm{~dB}$ if the amplitude error is eliminated, fitting well with the measured HR as well as the Monte Carlo simulation results. This also suggests that phase error can indeed be the limitation now.

\section{E. Digitally Enhanced $H R$}

Consider now the digitally enhanced HR architecture. The harmonic rejection for the third harmonic image versus LO tuning range (0.4 to $0.9 \mathrm{GHz}$ ) was measured, see Fig. 23. At the receiver input, the desired signal RF power was $-66.1 \mathrm{dBm}$ and the harmonic image RF power was $-20.1 \mathrm{dBm}$. The analog HR mixer provides more than $36 \mathrm{~dB}$ HR for the third harmonic image, which is higher than the $32.4 \mathrm{~dB}$ predicted by Table I. We attribute this difference to the finite output impedance of the three LNTAs. Thus, the effective weighting of the 2:3:2 ratio is closer to the ideal $1: \sqrt{2}: 1$, resulting in a higher measured HR.

Given a SIR of $-46 \mathrm{~dB}$ at RF, the digital AIC increases the harmonic rejection provided by the analog $\mathrm{HR}$ mixer from $36 \mathrm{~dB}$ to over $80 \mathrm{~dB}$ across the entire LO tuning range. The HR measurements are calculated based on the difference in power between the desired signal and the harmonic image. At the output
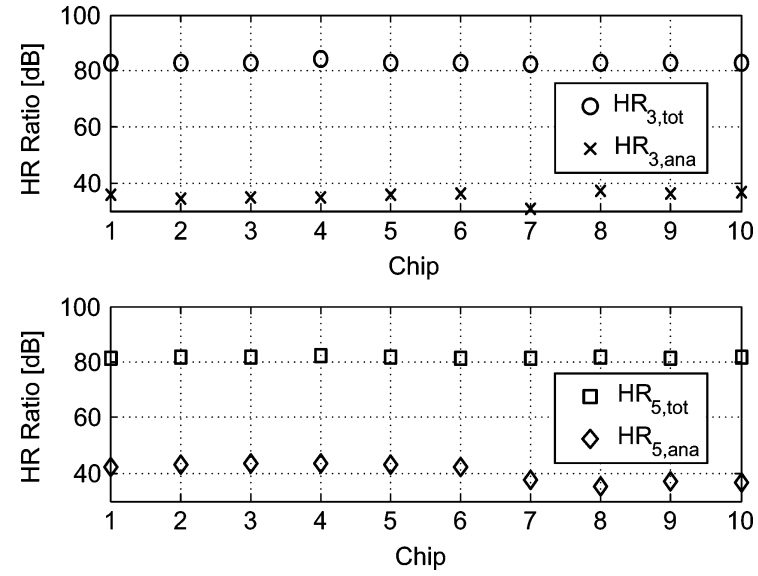

Fig. 24. The measured third and fifth order HR of the analog stage and combined stages, for 10 randomly selected chips, at $800 \mathrm{MHz}$ LO (digitally enhanced HR).

of the digital canceller, the harmonic image is below the noise floor. Instead of the harmonic image power, the noise floor was taken. Thus, the actual HR is greater than what is shown in Fig. 23.

A second indicator that the HR is higher comes from the SIR of the interference estimate, $\mathrm{v}(\mathrm{n})$, which was measured to be over $52 \mathrm{~dB}$ (limited by noise floor of equipment) across the entire LO tuning range. Given (5), the (theoretical) SIR at the output of the canceller is also $52 \mathrm{~dB}$. The power ratio between the desired signal and the harmonic image (at RF) is $-46 \mathrm{~dB}$, which makes the theoretical HR greater than $98 \mathrm{~dB}$ ! Unfortunately, the height of the noise floor at the output of the canceller, which is largely determined by the quantization noise of the A/D board, prevents this to be verified.

The third and fifth order harmonic rejection for multiple (randomly selected) chips is shown in Fig. 24. The desired signal RF power was $-66.1 \mathrm{dBm}$ at $800 \mathrm{MHz} \mathrm{LO}$. The RF power of the third and fifth order harmonic images was $-20.1 \mathrm{dBm}$. The results show more than $36 \mathrm{~dB}$ of analog harmonic rejection and more than $80 \mathrm{~dB}$ of combined harmonic rejection, for all chips. Thus, the digitally enhanced AIC technique performs well under varying mismatch conditions.

To show the effectiveness of the AIC technique against a modulated interferer, an FM modulated third harmonic image interferer was applied to the system. Fig. 25 shows the baseband spectrum of the received signal (top), r(n), and the AIC output (bottom), e(n).

In the received signal (Fig. 25, top), the third harmonic image signal at $-1.25 \mathrm{MHz}$ (baseband) causes interference to a (sinusoidal) desired signal at $-1.45 \mathrm{MHz}$ (baseband). The I/Q imbalance image of the third harmonic interferer is also visible at $+1.25 \mathrm{MHz}$ (baseband).

At the output of the canceller (Fig. 25, bottom), the third harmonic interferer is below the noise floor, which is a suppression of more than $40 \mathrm{~dB}$. Assuming at least $36 \mathrm{~dB}$ of analog harmonic rejection, the combined harmonic rejection is thus more than $76 \mathrm{~dB}$. This is less than $80 \mathrm{~dB}$ because the FM modulated interferer was weaker than the sinusoidal interferer used during the previous measurements. 


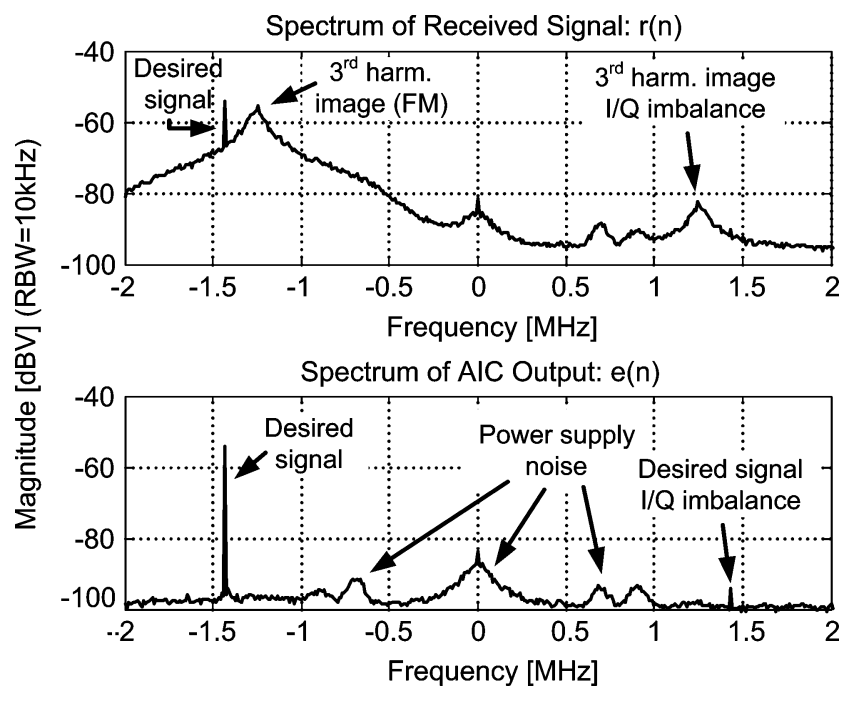

Fig. 25. (top) The measured baseband spectrum of the (discrete-time) received signal, $r(n)$, and (bottom) the output of the canceller, e(n).

Fig. 25 also shows signals which are caused by ground loop problems and spurs emanating from the switching power supply of the PC, which housed the A/D converter board.

Note that the I/Q imbalance image of the third harmonic interferer, see Fig. 25 (top), is suppressed to below the noise floor, revealing the I/Q imbalance image of the desired signal, see Fig. 25 (bottom), at $+1.45 \mathrm{MHz}$ (baseband).

After convergence of the LMS algorithm, which takes around 50000 samples $(12.5 \mathrm{~ms})$, the equalizer coefficients were $w_{1}=$ $0.4852-j \cdot 0.0086$ and $w_{2}=0.0221+j \cdot 0.0122$.

The measurements show that the digitally enhanced HR approach is indeed a powerful one; it produces unprecedented HR figures, irrespective of (small) analog mismatches that exist in the analog front-end.

\section{F. Comparing the Alternatives}

The two-stage polyphase HR implemented in analog approach helps both third and fifth HR via improved amplitude accuracy and achieves a minimum rejection of $60 \mathrm{~dB}$ and $64 \mathrm{~dB}$ respectively. The digitally enhanced HR based on AIC algorithm consistently shows more than $80 \mathrm{~dB}$ of $\mathrm{HR}$ for a single harmonic image (either the third or the fifth) by correcting both amplitude and phase of that harmonic image. The other harmonic image is rejected by at least $36 \mathrm{~dB}$, not improved from the analog first stage. They share a similar limitation on even-order HR.

On the implementation level, compared to the two-stage polyphase $\mathrm{HR}$, the digitally enhanced $\mathrm{HR}$ architecture requires two additional A/D converters, which may increase the power considerably. Fortunately, the converters for $x_{45}$ and $x_{135}$ (Fig. 9) may be switched off when the analog HR stages can provide enough harmonic rejection.

Table II summarizes the results and properties of the two alternative approaches.

\section{G. Performance Summary and Benchmark}

Table III summarizes the measured performance. As a benchmark, Table IV shows a comparison to other recently published
TABLE II

COMParison of Two Alternative HR TeChNiques Robust to Mismatch

\begin{tabular}{|c|c|c|}
\hline & Analog 2-stage & Digital AIC \\
\hline Rej. strongest & $>60 \mathrm{~dB}$ & $>80 \mathrm{~dB}$ a) \\
\hline Rej. other odd & $>64 \mathrm{~dB}$ & $>36 \mathrm{~dB}$ \\
\hline Rej. even & $>62 \mathrm{~dB}$ & $>64 \mathrm{~dB}$ \\
\hline Power front-end & $\begin{array}{c}50 \mathrm{~mA} @ 1.2 \mathrm{~V} \\
\text { (excl. ADCs) }\end{array}$ & $\begin{array}{c}44 \mathrm{~mA} @ 1.2 \mathrm{~V} \\
\text { (excl. ADCs) }\end{array}$ \\
\hline $\begin{array}{c}\text { Power DSP } \\
\text { (100 MS/s) }\end{array}$ & N/A & $\begin{array}{c}<8.5 \mathrm{~mA} @ 1.2 \mathrm{~V} \\
\text { (simulated) }\end{array}$ \\
\hline \# ADCs & 2 & 4 \\
\hline
\end{tabular}

a) If one harmonic interference image band is dominating.

TABLE III

Summary of MEASUREd Key PERformance

\begin{tabular}{|c|c|c|}
\hline \multicolumn{2}{|c|}{ LO Frequency } & 0.4 to $0.9 \mathrm{GHz}$ \\
\hline \multicolumn{2}{|c|}{ Gain } & $34.4 \mathrm{~dB} \pm 0.2 \mathrm{~dB}$ \\
\hline \multicolumn{2}{|c|}{ DSB NF } & $4 \mathrm{~dB} \pm 0.5 \mathrm{~dB}$ \\
\hline \multicolumn{2}{|c|}{$S_{11}<-10 d B$} & $80 \mathrm{MHz}$ to $5.5 \mathrm{GHz}$ \\
\hline \multicolumn{2}{|c|}{-3dB RF Bandwidth } & Up to $6 \mathrm{GHz}$ \\
\hline \multicolumn{2}{|c|}{ In/Out-of-band IIP3 } & $+3.5 \mathrm{dBm} /+16 \mathrm{dBm}$ \\
\hline \multicolumn{2}{|c|}{ In/Out-of-band IIP2 } & $+46 \mathrm{dBm} /+56 \mathrm{dBm}$ \\
\hline \multicolumn{2}{|c|}{-3dB IF Bandwidth } & $12 \mathrm{MHz}$ \\
\hline \multicolumn{2}{|c|}{$1 / \mathrm{f}$ noise } & $30 \mathrm{kHz}$ corner \\
\hline \multirow{2}{*}{$\begin{array}{l}\text { Harmonic } \\
\text { Rejection }\end{array}$} & Analog 2-stage & $2^{\text {nd }}-6^{\text {th }}:>60 \mathrm{~dB}$ \\
\hline & Digital AIC & 1 strongest: $>80 \mathrm{~dB}$ \\
\hline \multicolumn{2}{|c|}{ Technology } & $65 \mathrm{~nm}$ CMOS \\
\hline \multicolumn{2}{|c|}{ Supply Voltage } & $1.2 \mathrm{~V}$ \\
\hline \multicolumn{2}{|c|}{ DC Current } & $50 \mathrm{~mA}$ max. \\
\hline
\end{tabular}

wideband receivers with HR. Since it is difficult to characterize the exact overhead of the part implemented in software (digital AIC), here we only compare the part implemented on chip (Fig. 4) to other work.

There are two outstanding parameters of this work, i.e., linearity and harmonic rejection. Comparing all work including an LNA, [2], [4], and [33] shows an IIP3 around $-15 \mathrm{dBm}$ while this work shows an IIP3 of $+3.5 \mathrm{dBm}$ and a competitive NF. The OB IIP3 of our work is even higher $(+16 \mathrm{dBm})$, but we did not find a good way to benchmark it. For HR, only [32] and [33] reported numbers comparable to this work. However, [32] only reported results from one chip while consuming large power due to a different structure of the HR mixer. [33] reported results for 10 chips, but relying on hand calibration, and the calibration is only effective for either third or fifth HR but not for both at the same time. Thus, we conclude that our design has both good linearity and good HR at moderate power consumption, thanks to the proposed techniques.

\section{CONCLUSION}

This paper identified out-of-band (OB) nonlinearity and harmonic mixing as two main problems for out-of-band interference (OBI), and proposed solutions to reduce their effects. First, 
TABLE IV

BENCHMARK WITH OTHER RECENT WORK

\begin{tabular}{|c|c|c|c|c|c|c|c|c|c|c|}
\hline Benchmark & $\begin{array}{l}\text { CMOS } \\
\text { Tech. }\end{array}$ & $\begin{array}{l}\text { Freq. } \\
(\mathrm{MHz})\end{array}$ & $\begin{array}{l}\text { Gain } \\
(\mathrm{dB})\end{array}$ & $\begin{array}{l}\mathrm{NF} \\
(\mathrm{dB})\end{array}$ & $\begin{array}{c}\text { IIP3 } \\
(\mathrm{dBm})\end{array}$ & $\begin{array}{l}\text { HR3 } \\
\text { (dB) }\end{array}$ & $\begin{array}{l}\text { HR5 } \\
(\mathrm{dB})\end{array}$ & Chip \# & Cal. & $\begin{array}{l}\text { Power } \\
(\mathrm{mW})\end{array}$ \\
\hline \multirow[t]{2}{*}{ This Work } & \multirow[t]{2}{*}{$65 \mathrm{~nm}$} & \multirow{2}{*}{$\begin{array}{c}400- \\
900 \text { a) }\end{array}$} & 34 & 4 & +3.5 & 60 & 64 & $40 \mathrm{~min}$. & No & 60 \\
\hline & & & \multicolumn{8}{|c|}{ LNA + Mixer + Divider } \\
\hline \multirow{2}{*}{$\begin{array}{l}\text { Bagheri, } \\
\text { JSSC06 [2] }\end{array}$} & \multirow[t]{2}{*}{$90 \mathrm{~nm}$} & \multirow{2}{*}{$\begin{array}{l}800- \\
5000\end{array}$} & 36 & 5 & $-15^{b)}$ & 38 & 40 & 1 & No & 51 \\
\hline & & & \multicolumn{8}{|c|}{ LNA + Mixer + Divider, performance at $900 \mathrm{MHz}$} \\
\hline \multirow{2}{*}{$\begin{array}{l}\text { Lerstaveesin, } \\
\text { JSSC08 [4] }\end{array}$} & \multirow[t]{2}{*}{$180 \mathrm{~nm}$} & \multirow{2}{*}{$\begin{array}{l}48- \\
860\end{array}$} & 83 & 4 to 7 & -14 & $42^{c)}$ & $?$ & 1 & No & $468^{c)}$ \\
\hline & & & \multicolumn{8}{|c|}{ LNA + Mixer + BB filter + VGA + ADC + PLL } \\
\hline \multirow{2}{*}{$\begin{array}{c}\mathrm{Ru}, \\
\text { ISSCC08 [16] }\end{array}$} & \multirow[t]{2}{*}{$65 \mathrm{~nm}$} & \multirow{2}{*}{$\begin{array}{l}200- \\
900\end{array}$} & 2 & 19 & +11 & 41 & 44 & 10 av. & No & 19 \\
\hline & & & \multicolumn{8}{|c|}{ No LNA, only mixer \& multiphase clock } \\
\hline \multirow{2}{*}{\begin{tabular}{c|} 
Maxim, \\
ISSCC08 [32]
\end{tabular}} & \multirow[t]{2}{*}{$130 \mathrm{~nm}$} & \multirow{2}{*}{$\begin{array}{c}40- \\
1000\end{array}$} & $?$ & 16 & +20 & 63 & 64 & 1 & No & 450 \\
\hline & & & \multicolumn{8}{|c|}{ No LNA, only mixer \& multiphase clock } \\
\hline \multirow{2}{*}{$\begin{array}{c}\text { Cha, } \\
\text { MWCL08 [33] }\end{array}$} & \multirow[t]{2}{*}{$180 \mathrm{~nm}$} & \multirow{2}{*}{$\begin{array}{l}48- \\
862\end{array}$} & 35 & 8 & -15 & 72 & 45 & $10 \mathrm{~min}$. & Yes & 189 \\
\hline & & & \multicolumn{8}{|c|}{ LNA + Mixer + Divider } \\
\hline
\end{tabular}

a) Only LO frequency range, while the measured RF bandwidth is up to $6 \mathrm{GHz}$.

b) IIP3 is derived for the full-gain setting which is required to match the $5 \mathrm{~dB} N F$

c) Both the HR3 and the power data exclude the contribution of RF tracking filter.

OB nonlinearity can be improved by implementing low-pass filtering, simultaneously with voltage gain only after downconversion, to improve the OB IIP3 and the desensitization point due to blockers. Second, two "iterative" harmonic-rejection (HR) techniques are presented to reduce harmonic mixing in a way which is robust to mismatch. An analog two-stage polyphase HR concept is proposed to greatly enhance the amplitude accuracy for both third and fifth harmonics so that the total amplitude error becomes product of errors. Alternatively, digitally enhanced HR based on adaptive interference cancelling (AIC) can be applied to improve HR of the analog first-stage further by correcting both amplitude and phase errors for one dominant harmonic, either third or fifth. To guarantee a mismatch-robust HR for both analog and digital approaches, a simple but accurate ring counter is presented to generate the multiphase clocks driving the HR mixer.

We have verified the proposed techniques via a SDR receiver in $65 \mathrm{~nm} \mathrm{CMOS,} \mathrm{with} \mathrm{RF} \mathrm{bandwidth} \mathrm{up} \mathrm{to} 6 \mathrm{GHz}$ and 8-phase LO frequency up to $0.9 \mathrm{GHz}$ (master clock up to $7.2 \mathrm{GHz}$ ). The $1 \mathrm{~dB}$ compression point is $-22 \mathrm{dBm}$ while the maximum $1 \mathrm{~dB}$ desensitization point is more than $0 \mathrm{dBm}$, showing the low-pass blocker filtering is effective. In terms of IIP $3,+16 \mathrm{dBm}$ for OBI is measured without fine tuning for sufficient frequency spacing, e.g., $\mathrm{LO}$ at $819 \mathrm{MHz}$ while two-tone at $1.61 \mathrm{G}$ and $2.40 \mathrm{GHz}$, versus an in-band IIP3 of $+3.5 \mathrm{dBm}$. Without any trimming or calibration, the two-stage polyphase HR technique achieves $60 \mathrm{~dB}$ minimum HR ratio at $0.8 \mathrm{GHz}$ LO for both third and fifth harmonics over 40 randomly selected chips, and all even-order HR ratios are measured above $60 \mathrm{~dB}$ as well. The digital AIC HR achieves a steady $80 \mathrm{~dB}$ HR for either third or fifth harmonic for 10 chips, indicating the power of adaptive digital techniques to solve analog problems.

\section{APPENDIX}

Effect of Random Amplitude and Phase Errors to Harmonic Rejection: This Appendix aims at estimating the HR ratio and its sensitivity to amplitude and phase errors. These effects have been partly considered in [2] and [15], however, the statistical nature of mismatch and the effect of using balanced RF or balanced LO have not been included so far. We will also consider the effect of LO duty cycle "d".

Suppose we have three signal paths to the output (as in Fig. 3 to Fig. 7) and the signals are represented by vectors as in Figs. 3(b) and 7. The resulted first and third harmonics can be respectively written as

$$
\begin{aligned}
& H_{1}=R_{H 1} \cdot\left[A_{1} \cos \varphi_{1}+\sqrt{2} \cos 0^{\circ}+A_{2} \cos \varphi_{2}\right] \\
& H_{3}=R_{H 3} \cdot\left[A_{1} \cos \left(3 \varphi_{1}\right)+\sqrt{2} \cos 0^{\circ}+A_{2} \cos \left(3 \varphi_{2}\right)\right]
\end{aligned}
$$

where $R_{H 1}$ and $R_{H 3}$ are the Fourier series coefficients of a pulse-wave LO with duty cycle " $\mathrm{d}$ "

$$
\begin{aligned}
R_{H 1} & =\frac{2}{\pi} \sin (\pi \cdot d), \quad R_{H 3}=\frac{2}{3 \pi} \sin (3 \pi \cdot d), \\
A_{1} & =\left(1+\Delta A_{1}\right), \quad A_{2}=\left(1+\Delta A_{2}\right), \\
\varphi_{1} & =\left(45^{\circ}+\Delta \varphi_{1}\right), \quad \varphi_{2}=\left(-45^{\circ}+\Delta \varphi_{2}\right) .
\end{aligned}
$$

If $\Delta A_{1}, \Delta A_{2}, \Delta \varphi_{1}, \Delta \varphi_{2}$ are small and uncorrelated, we can approximate the variance in $H_{3}$ as shown in (A.2), the first equation at the top of the next page. If $\sigma_{A 1}=\sigma_{A 2}=\sigma_{A}$ and $\sigma_{\varphi 1}=\sigma_{\varphi 2}=\sigma_{\varphi}$, then results (A.3), the second equation shown at the top of the next page. Since $H_{1} \approx 2 \sqrt{2} \cdot R_{H 1}$, taking the ratio, we obtain

$$
\begin{aligned}
\left(\frac{\sigma_{H 3}^{2}}{H_{1}^{2}}\right) & \approx \frac{R_{H 3}^{2} \cdot\left[\sigma_{A}^{2}+9 \sigma_{\varphi}^{2}\right]}{8 R_{H 1}^{2}} \\
& =\frac{\sin ^{2}(3 \pi \cdot d)}{\sin ^{2}(\pi \cdot d)}\left[\left(\frac{\sigma_{A}}{6 \sqrt{2}}\right)^{2}+\left(\frac{\sigma_{\varphi}}{2 \sqrt{2}}\right)^{2}\right] .
\end{aligned}
$$

Please note that $\sigma_{A}$ is the standard deviation of amplitude error in percentage and $\sigma_{\varphi}$ is the standard deviation of phase error in radians.

For a double-balanced HR mixer, which creates the output during one half period from 0 to $\mathrm{T} / 2$ with the positive-sign RF-LNTA path then the other half from $\mathrm{T} / 2$ to $\mathrm{T}$ with the negative-sign RF-LNTA path, the first harmonic adds up in amplitude while the third harmonic adds up in power (as the 


$$
\sigma_{H 3}^{2} \approx R_{H 3}^{2} \cdot\left[\left(\frac{\partial H_{3}}{\partial A_{1}}\right)^{2} \cdot \sigma_{A 1}^{2}+\left(\frac{\partial H_{3}}{\partial A_{2}}\right)^{2} \cdot \sigma_{A 2}^{2}+\left(\frac{\partial H_{3}}{\partial \varphi_{1}}\right)^{2} \cdot \sigma_{\varphi 1}^{2}+\left(\frac{\partial H_{3}}{\partial \varphi_{2}}\right)^{2} \cdot \sigma_{\varphi 2}^{2}\right]
$$

$$
\sigma_{H 3}^{2} \approx R_{H 3}^{2} \cdot\left[2 \cos ^{2}\left(3 \cdot 45^{\circ}\right) \cdot \sigma_{A}^{2}+18 \sin ^{2}\left(3 \cdot 45^{\circ}\right) \cdot \sigma_{\varphi}^{2}\right]=R_{H 3}^{2} \cdot\left[\sigma_{A}^{2}+9 \sigma_{\varphi}^{2}\right]
$$

error is uncorrelated between two half periods for both amplitude and phase). Therefore, the ratio is improved by $3 \mathrm{~dB}$ for a double-balanced HR mixer compared to (A.4), i.e.,

$$
\left(\frac{\sigma_{H 3}^{2}}{H_{1}^{2}}\right)_{\mathrm{diff}} \approx \frac{\sin ^{2}(3 \pi \cdot d)}{\sin ^{2}(\pi \cdot d)}\left[\left(\frac{\sigma_{A}}{12}\right)^{2}+\left(\frac{\sigma_{\varphi}}{4}\right)^{2}\right] .
$$

If the duty cycle of the $\mathrm{LO}$ is $50 \%$ or $25 \%$, i.e., $d=0.5$ or 0.25 , we get

$$
\left(\frac{\sigma_{H 3}^{2}}{H_{1}^{2}}\right)_{\mathrm{diff}, 50 \%} \approx\left(\frac{\sigma_{A}}{12}\right)^{2}+\left(\frac{\sigma_{\varphi}}{4}\right)^{2} .
$$

If there is no amplitude error, $50 \%$ or $25 \%$ duty cycle results in a $3 \sigma-\mathrm{HR} 3$ of $70 \mathrm{~dB}$ if $\sigma_{\varphi}=0.024^{\circ}$. If the duty cycle is $1 / 8$, i.e., $d=0.125$, as in our case, we get

$$
\left(\frac{\sigma_{H 3}^{2}}{H_{1}{ }^{2}}\right)_{\mathrm{diff}, 12.5 \%} \approx 5.8 \cdot\left[\left(\frac{\sigma_{A}}{12}\right)^{2}+\left(\frac{\sigma_{\varphi}}{4}\right)^{2}\right] .
$$

Without amplitude error, the $3 \sigma$-HR3 is now $62 \mathrm{~dB}$.

A similar derivation for fifth order HR of a double-balanced HR mixer renders

$$
\left(\frac{\sigma_{H 5}^{2}}{H_{1}^{2}}\right)_{\mathrm{diff}} \approx \frac{\sin ^{2}(5 \pi \cdot d)}{\sin ^{2}(\pi \cdot d)} \cdot\left[\left(\frac{\sigma_{A}}{20}\right)^{2}+\left(\frac{\sigma_{\varphi}}{4}\right)^{2}\right]
$$

where the phase term $\sigma_{\varphi}$ would have been multiplied by 5 in (A.4) due to the 5-times phase shift of $H_{5}$ compared to $H_{1}$. Nevertheless, without amplitude errors, this leads to the same numerical result $\left(\sigma_{\varphi}=0.024^{\circ}\right)$ : a $3 \sigma$-HR 5 of $62 \mathrm{~dB}$ for $1 / 8$ duty cycle LO.

\section{ACKNOWLEDGMENT}

The authors would like to thank Freeband Communications for research funding and NXP Semiconductors for chip fabrication, especially for the help from H. Brekelmans and D. Leenaerts. The authors also thank R. Dutta, X. Gao, and S. M. Louwsma for useful discussions on the clock generator design, and G. J. M. Wienk for his contribution to part of the layout and excellent assistance, as well as H. de Vries for his valuable support.

\section{REFERENCES}

[1] V. Giannini et al., "A $2 \mathrm{~mm} 0.1$ G-to-5 GHz SDR receiver in $45 \mathrm{~nm}$ digital CMOS," in IEEE ISSCC Dig. Tech. Papers, Feb. 2009, pp. 408-409.

[2] R. Bagheri et al., "An 800-MHz-6-GHz software-defined wireless receiver in 90-nm CMOS," IEEE J. Solid-State Circuits, vol. 41, no. 12, pp. 2860-2876, Dec. 2006.
[3] F. Gatta et al., "An embedded 65 nm CMOS low-IF 48 MHz-to- $1 \mathrm{GHz}$ dual tuner for DOCSIS 3.0," in IEEE ISSCC Dig. Tech. Papers, Feb. 2009, pp. 122-123.

[4] S. Lerstaveesin, M. Gupta, D. Kang, and B.-S. Song, "A 48-860 MHz CMOS low-IF direct-conversion DTV tuner," IEEE J. Solid-State Circuits, vol. 43, no. 9, pp. 2013-2024, Sep. 2008.

[5] S. C. Blaakmeer, E. Klumperink, D. M. W. Leenaerts, and B. Nauta, "The BLIXER, a wideband balun-LNA-I/Q-mixer topology," IEEE J. Solid-State Circuits, vol. 43, no. 12, pp. 2706-2715, Dec. 2008.

[6] D. Leenaerts et al., "A $65 \mathrm{~nm}$ CMOS inductorless triple-band-group WiMedia UWB PHY,” in IEEE ISSCC Dig. Tech. Papers, Feb. 2009, pp. 410-411.

[7] D. Manstretta, N. Laurenti, and R. Castello, "A reconfigurable narrowband MB-OFDM UWB receiver architecture," IEEE Trans. Circuits Syst. II: Expr. Briefs, vol. 55, no. 4, pp. 324-328, Apr. 2008.

[8] T. Sowlati et al., "Single-chip multiband WCDMA/HSDPA/HSUPA/ EGPRS transceiver with diversity receiver and 3G DIGRF interface without SAW filters in transmitter/3G receiver paths," in IEEE ISSCC Dig. Tech. Papers, Feb. 2009, pp. 116-117.

[9] O. Gaborieau et al., "A SAW-less multiband WEDGE receiver," in IEEE ISSCC Dig. Tech. Papers, Feb. 2009, pp. 114-115.

[10] B. Razavi, $R F$ Microelectronics. Upper Saddle River, NJ: Prentice Hall, 1998.

[11] V. Aparin and L. E. Larson, "Modified derivative superposition method for linearizing FET low-noise amplifiers," IEEE Trans. Microw. Theory Tech., vol. 53, no. 2, pp. 571-581, Feb. 2005.

[12] W.-H. Chen, G. Liu, B. Zdravko, and A. M. Niknejad, "A highly linear broadband CMOS LNA employing noise and distortion cancellation," IEEE J. Solid-State Circuits, vol. 43, no. 5, pp. 1164-1176, May 2008.

[13] E. A. M. Klumperink and B. Nauta, "Systematic comparison of HF CMOS transconductors," IEEE Trans. Circuits Syst. II, Analog Digit. Signal Process., vol. 50, no. 10, pp. 728-741, Oct. 2003.

[14] H. Darabi, "A blocker filtering technique for SAW-less wireless receivers," IEEE J. Solid-State Circuits, vol. 42, no. 12, pp. 2766-2773, Dec. 2007.

[15] J. A. Weldon et al., "A 1.75-GHz highly integrated narrowband CMOS transmitter with harmonic-rejection mixers," IEEE J. Solid-State Circuits, vol. 36, no. 12, pp. 2003-2015, Dec. 2001.

[16] Z. Ru, E. Klumperink, and B. Nauta, "A discrete-time mixing receiver architecture with wideband harmonic rejection," in IEEE ISSCC Dig. Tech. Papers, Feb. 2008, pp. 322-616.

[17] Z. Ru, E. Klumperink, G. Wienk, and B. Nauta, "A software-defined radio receiver architecture robust to out-of-band interference," in IEEE ISSCC Dig. Tech. Papers, Feb. 2009, pp. 230-231.

[18] Z. Ru, E. Klumperink, B. Nauta, and H. Brekelmans, "Polyphase harmonic rejection mixer," Netherlands Patent EP 091000095.0, Filed, Feb. 2009.

[19] N. A. Moseley, Z. Ru, E. Klumperink, and B. Nauta, "A 400-to-900 $\mathrm{MHz}$ receiver with dual-domain harmonic rejection exploiting adaptive interference cancellation," in IEEE ISSCC Dig. Tech. Papers, Feb. 2009, pp. 232-233.

[20] N. A. Moseley, E. Klumperink, and B. Nauta, "A two-stage approach to harmonic rejection mixing using blind interference cancellation," IEEE Trans. Circuits Syst. II: Expr. Briefs, vol. 55, no. 10, pp. 966-970, Oct. 2008.

[21] Z. Ru, "Frequency translation techniques for interference-robust software-defined radio receivers," Ph.D. dissertation, Univ. of Twente, Enschede, The Netherlands, 2009. 
[22] W. Redman-White and D. M. W. Leenaerts, "1/f noise in passive CMOS mixers for low and zero if integrated receivers," in Proc. 27th ESSCIRC, Sep. 18-20, 2001, pp. 41-44.

[23] M. Valla et al., "A 72-mW CMOS 802.11a direct conversion front-end with 3.5-dB NF and 200-kHz 1/f noise corner," IEEE J. Solid-State Circuits, vol. 40, no. 4, pp. 970-977, Apr. 2005.

[24] D. Kaczman et al., "A single-chip 10-Band WCDMA/HSDPA 4-Band GSM/EDGE SAW-less CMOS receiver with DigRF 3G interface and +90 dBm IIP2," IEEE J. Solid-State Circuits, vol. 44, no. 3, pp. 718-739, Mar. 2009.

[25] S. Haykin, Adaptive Filter Theory. Upper Saddle River, NJ: Prentice Hall, 2002.

[26] B. Widrow et al., "Adaptive noise cancelling: Principles and applications," Proc. IEEE, vol. 63, no. 12, pp. 1692-1716, Dec. 1975.

[27] M. Valkama, M. Renfors, and V. Koivunen, "Advanced methods for I/Q imbalance compensation in communication receivers," IEEE Trans. Signal Process., vol. 49, no. 10, pp. 2335-2344, Oct. 2001.

[28] F. Bruccoleri, E. A. M. Klumperink, and B. Nauta, "Wide-band CMOS low-noise amplifier exploiting thermal noise canceling," IEEE J. SolidState Circuits, vol. 39, no. 2, pp. 275-282, Feb. 2004.

[29] B. Razavi, Design of Analog CMOS Integrated Circuits. New York: McGraw-Hill, 2000.

[30] T. H. Lee, The Design of CMOS Radio-Frequency Integrated Circuits, 2 ed. Cambridge, U.K.: Cambridge Univ. Press, 2003.

[31] S. Rabii and B. A. Wooley, "A 1.8-V digital-audio sigma-delta modulator in 0.8- $\mu$ m CMOS," IEEE J. Solid-State Circuits, vol. 32, no. 6, pp. 783-796, Jun. 1997.

[32] A. Maxim et al., "A DDFS driven mixing-DAC with image and harmonic rejection capabilities," in IEEE ISSCC Dig. Tech. Papers, Feb. 2008, pp. 372-373.

[33] H. Cha, S. Song, H. Kim, and K. Lee, "A CMOS harmonic rejection mixer with mismatch calibration circuitry for digital TV tuner applications," IEEE Trans. Microw. Wireless Compon. Lett., vol. 18, no. 9, pp. 617-619, Sep. 2008.

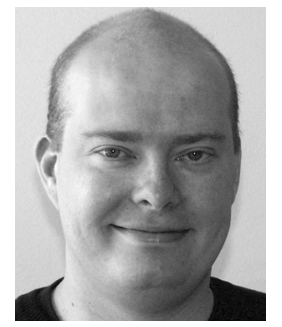

Niels A. Moseley was born on December 9, 1973, in Alphen aan de Rijn, The Netherlands. He received the M.Sc. degree in electrical engineering from the University of Twente in 2004 and is now pursuing the $\mathrm{Ph} . \mathrm{D}$. in the same field. His interests include digital signal processing for audio and wireless applications.

The subject of his Ph.D. work is performance enhancement of analog electronics using digital adaptive techniques. The main focus is on harmonic rejection enhancement techniques for software-defined radio applications.

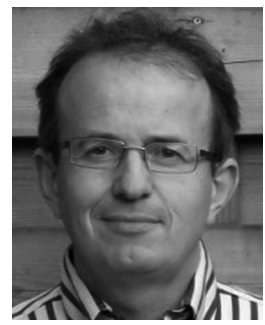

Eric A. M. Klumperink (M'98-SM'06) was born on April 4, 1960, in Lichtenvoorde, The Netherlands. He received the B.Sc. degree from HTS, Enschede, The Netherlands, in 1982. After a short period in industry, he joined the Faculty of Electrical Engineering of the University of Twente (UT) in Enschede, in 1984, participating in analog CMOS circuit design and research. This resulted in several publications and a Ph.D. thesis, in 1997 (Transconductance Based CMOS Circuits). After his Ph.D., he started working on RF CMOS circuits, and he is currently an Associate Professor at the IC-Design Laboratory, which participates in the CTIT Research Institute (UT).

He holds several patents and has authored and co-authored more than 80 journal and conference papers. In 2006 and 2007, he served as Associate Editor for IEEE TRANSACTIONS ON CIRCUITS AND SYSTEMS II and since 2008 for IEEE TRANSACTIONS ON CIRCUITS AND SYSTEMSI.

Dr. Klumperink was a co-recipient of the ISSCC 2002 Van Vessem Outstanding Paper Award.

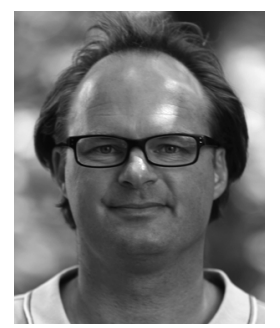

Bram Nauta (M'91-SM'03-F'07) was born in Hengelo, The Netherlands, in 1964. In 1987 he received the M.Sc degree (cum laude) in electrical engineering from the University of Twente, Enschede, The Netherlands. In 1991 he received the $\mathrm{Ph}$.D. degree from the same university on the subject of analog CMOS filters for very high frequencies.

In 1991 he joined the Mixed-Signal Circuits and Systems Department of Philips Research, Eindhoven, The Netherlands, where he worked on high-speed AD converters and analog key modules. In 1998 he returned to the University of Twente, as full Professor heading the IC Design group, which is part of the CTIT Research Institute. His current research interest is high-speed analog CMOS circuits. He is also a part-time consultant in industry, and in 2001 he co-founded Chip Design Works.

His Ph.D. thesis was published as a book: Analog CMOS Filters for Very High Frequencies (Springer, 1993) and he received the Shell Study Tour Award for his Ph.D. work. From 1997 until 1999 he served as Associate Editor of IEEE TRANSACtions on Circuits and Systems II-ANALOG AND Digital SigNAL Processing. After this, he served as Guest Editor, Associate Editor (2001-2006), and from 2007 as Editor-in-Chief for the IEEE JOURNAL OF SOLID-STATE CIRCUITS. He is also a member of the technical program committees of the IEEE International Solid State Circuits Conference (ISSCC), the European Solid State Circuits Conference (ESSCIRC), and the Symposium on VLSI Circuits. He is a co-recipient of the ISSCC 2002 Van Vessem Outstanding Paper Award, a Distinguished Lecturer of the IEEE, an elected member of IEEE SSCS AdCom and an IEEE Fellow. 